\title{
Impaired angiogenesis and extracellular matrix metabolism in autosomal-dominant hyper-IgE syndrome
}

\author{
Natalia I. Dmitrieva, ${ }^{1}$ Avram D. Walts, ${ }^{1}$ Dai Phuong Nguyen, ${ }^{1}$ Alex Grubb, ${ }^{1}$ Xue Zhang, ${ }^{2}$ Xujing Wang, ${ }^{2}$ Xianfeng Ping, ${ }^{1}$ Hui Jin, ${ }^{1}$ \\ Zhen Yu, ${ }^{1}$ Zu-Xi Yu, ${ }^{3}$ Dan Yang, ${ }^{1}$ Robin Schwartzbeck, ${ }^{1}$ Clifton L. Dalgard, ${ }^{4,5,6}$ Beth A. Kozel, ${ }^{7}$ Mark D. Levin, ${ }^{7}$ Russell H. Knutsen, ${ }^{7}$ \\ Delong Liu, ${ }^{7}$ Joshua D. Milner, ${ }^{8}$ Diego B. López, ${ }^{8}$ Michael P. O'Connell, ${ }^{8}$ Chyi-Chia Richard Lee, ${ }^{9}$ Ian A. Myles, ${ }^{10}$ Amy P. Hsu, ${ }^{10}$ \\ Alexandra F. Freeman, ${ }^{10}$ Steven M. Holland, ${ }^{10}$ Guibin Chen, ${ }^{1}$ and Manfred Boehm ${ }^{1}$ \\ 'Laboratory of Cardiovascular Regenerative Medicine, Translational Vascular Medicine Branch, ${ }^{2}$ Bioinformatics and Systems Biology Core, and ${ }^{3}$ Pathology Core, National Heart, Lung, and Blood Institute \\ (NHLBI), NIH, Bethesda, Maryland, USA. ${ }^{4}$ Department of Anatomy, Physiology \& Genetics, ${ }^{5}$ The American Genome Center, and ${ }^{6}$ Collaborative Health Initiative Research Program, Uniformed Services \\ University of the Health Sciences, Bethesda, Maryland, USA. 'Laboratory of Vascular and Matrix Genetics, NHLBI, ${ }^{8}$ Laboratory of Allergic Diseases, National Institute of Allergy and Infectious Diseases \\ (NIAID), ' Laboratory of Pathology, Center for Cancer Research, National Cancer Institute (NCI), and ${ }^{10}$ Laboratory of Clinical Immunology and Microbiology, NIAID, NIH, Bethesda, Maryland, USA.
}

\begin{abstract}
There are more than 7000 described rare diseases, most lacking specific treatment. Autosomal-dominant hyper-lgE syndrome (AD-HIES, also known as Job's syndrome) is caused by mutations in STAT3. These patients present with immunodeficiency accompanied by severe nonimmunological features, including skeletal, connective tissue, and vascular abnormalities, poor postinfection lung healing, and subsequent pulmonary failure. No specific therapies are available for these abnormalities. Here, we investigated underlying mechanisms in order to identify therapeutic targets. Histological analysis of skin wounds demonstrated delayed granulation tissue formation and vascularization during skin-wound healing in AD-HIES patients. Clobal gene expression analysis in AD-HIES patient skin fibroblasts identified deficiencies in a STAT3-controlled transcriptional network regulating extracellular matrix (ECM) remodeling and angiogenesis, with hypoxia-inducible factor $1 \alpha$ (HIF-1 $\alpha$ ) being a major contributor. Consistent with this, histological analysis of skin wounds and coronary arteries from AD-HIES patients showed decreased HIF-1 $\alpha$ expression and revealed abnormal organization of the ECM and altered formation of the coronary vasa vasorum. Disease modeling using cell culture and mouse models of angiogenesis and wound healing confirmed these predicted deficiencies and demonstrated therapeutic benefit of HIF- $1 \alpha$-stabilizing drugs. The study provides mechanistic insights into AD-HIES pathophysiology and suggests potential treatment options for this rare disease.
\end{abstract}

\section{Introduction}

There are more than 7000 identified rare diseases that affect fewer than 1 person per 2000 and in most cases are caused by the altered function of a single gene (1). These diseases can not only be disastrous for the patients, but collectively exert a large impact on health care throughout the world (2). In spite of identification of genetic causes for many rare diseases, there are treatments for only about $6 \%$ of them (1).

Autosomal-dominant hyper-IgE syndrome (AD-HIES, also known as Job's syndrome) is a rare immunodeficiency affecting fewer than 1 in a million people worldwide $(3,4)$. In 2007 , mutations in the STAT3 gene were identified as the genetic cause of AD-HIES $(3,4)$. The multiple transcriptional targets with crucial roles in basic cellular functions and the widespread expression of STAT3 protein $(5,6)$ helped remove part of the mystery surrounding the multisystem presentation of $\mathrm{AD}$-HIES. In addition to multiple life-threatening infections, such as recurrent staphylococcal abscesses and

Authorship note: NID and ADW are co-first authors

Conflict of interest: The authors have declared that no conflict of interest exists. Copyright: (5) 2020, American Society for Clinical Investigation.

Submitted: December 9, 2019; Accepted: April 29, 2020; Published: June 29, 2020.

Reference information: / Clin Invest. 2020;130(8):4167-4181

https://doi.org/10.1172/JCl135490. pyogenic pneumonias starting early in childhood, the disease is accompanied by multiple abnormalities outside the immune system. As opposed to complete tissue healing experienced by otherwise healthy patients after pneumonia, aberrant healing often occurs in AD-HIES patients. This process is characterized by prolonged inflammation and the formation of chronically inflamed pneumatoceles that in turn attract secondary fungal and bacterial infections. Such cycles of recurrent infections and aberrant healing gradually destroy the lung parenchyma, leading to significant morbidity and mortality for AD-HIES patients (7-9). Additionally, skeletal and connective tissue abnormalities, including facial dysmorphism, osteoporosis, fractures with minor trauma, scoliosis, and joint hyperextensibility, are frequent in AD-HIES patients (10, 11). Vascular abnormalities include arterial tortuosity and abnormal dilatation and aneurysms of medium-sized arteries (11-14), all of which can lead to potentially fatal complications, such as myocardial infarction and subarachnoid hemorrhage $(15,16)$.

In spite of the identified genetic cause of the disease, the exact mechanisms of nonhematopoietic AD-HIES pathologies are not well understood. This prevents the development of specific therapies and limits treatment options to general antimicrobial prophylaxis and supportive multidisciplinary care rather than treatment for the underlying causes of the disease pathologies. Through optimization of preventative antimicrobial treatments, many AD-HIES 
patients live long enough for nonimmune complications to become a major cause of death $(10,16,17)$. In the current study, we investigate mechanisms underlying nonimmune abnormalities of AD-HIES in order to better understand the disease pathophysiology and find a promising target or targets for pharmacological therapy.

$\mathrm{AD}$-HIES's multisystem nature comes from the diverse functions of STAT3. STAT3 acts as a major hub that integrates and transduces signaling from more than 40 cytokines and growth factors and regulates transcription of multiple genes $(5,6,18)$. The fact that so many systems are affected makes it difficult to decide which pathway should be targeted for therapy. Specificity of outcomes is achieved by fine-tuning of relative level of expression and activity of the network members. Phenotypes with different degrees of overlap with AD-HIES immune and nonimmune features were discovered in patients carrying disactivating mutations in genes encoding receptors of cytokines upstream of STAT3, such as IL-11 receptor (IL-11R) (19), common GP130 cytokine receptor subunit (IL-6ST) (20, 21), and IL-6 receptor (IL-6R) (22) as well as mutations in ZNF341 regulating STAT3 transcription (23, 24). Several dozen heterozygous dominant negative mutations in the STAT3 gene that result in AD-HIES have been identified (18). The mutations are located in either the DNA-binding or the protein dimerization domains, resulting in a 1:1 mixture of WT and mutated proteins that allows for a residual normal function of about $20 \%$ to $30 \%$ of the dimers. Furthermore, the mutated protein is not functionally silent and can potentially interfere with signaling pathways in which STAT3 participates as a part of multiprotein complexes (25). Given these complexities, the exact consequences of the functional changes that the AD-HIES STAT3 mutations introduce into signaling networks are difficult to predict. To overcome this uncertainty and find promising therapeutic targets, we performed global gene-expression analyses that have allowed us to objectively identify pathways changed specifically by AD-HIES mutations in primary cells from AD-HIES patients. The analyses identified deficiencies in signaling related to angiogenesis, extracellular matrix (ECM) remodeling, and wound healing and predicted hypoxia-inducible factor $1 \alpha$ (HIF$1 \alpha)$ as a key mediator of these deficiencies. Given the morbidity and mortality associated with abnormal wound healing in patients with AD-HIES, these pathways seemed an ideal target to investigate for a clinically impactful therapy.

Here, we show that AD-HIES patients exhibit attenuated skinwound healing due to deficient neovascularization and delayed restoration of dermal collagen and have aberrant ECM composition of the coronary artery walls. We demonstrate that HIF-1 $\alpha-$ stabilizing drugs, already in the late stages of clinical development for more common diseases (26-28), substantially improve angiogenesis and wound healing in cell culture and mouse models of AD-HIES. The findings of this study yield insights into AD-HIES disease mechanisms and open up a promising treatment possibility for this rare disease.

\section{Results}

Delayed skin-wound healing in AD-HIES patients. Aberrant postinfection healing of the lung parenchyma leading to formation of pneumatoceles and frequent postsurgery complications experienced by patients with AD-HIES (7-9) indicate major deficiencies in processes involved in wound healing and tissue remodeling. In order to gain insights into mechanisms underlying these abnormalities, we developed a human skin-wound-healing model in which we analyzed healing of skin biopsy wounds created by removing cylindrical pieces of skin using a 2-mm diameter punch biopsy tool (Figure 1A and Supplemental Figure 1; supplemental material available online with this article; https://doi.org/10.1172/ JCI135490DS1). Histological examination of the traumatized tissue obtained by repeated 3-mm punch biopsy performed at day 7 after injury revealed histologic features consistent with a delayed wound-healing process in AD-HIES patients. In control patient wounds, abundant dermal collagen (highlighted by Masson's trichrome stain) was present within the wound granulation tissue. By comparison, at day 7, AD-HIES wounds were still composed of mostly poorly organized granulation tissue, with nearly absent or minimal amounts of dermal collagen (Figure 1, A-C, and Supplemental Figure 1, C and D). While control fibroblasts in active granulation tissue during wound healing formed abundant collagen fibers (Figure 1C) and were surrounded by a higher density of blood vessels (Figure 1, D and E, and Supplemental Figure 1B), AD-HIES fibroblasts formed less collagen (Figure 1C) and the granulation tissue contained many fewer blood vessels (Figure 1, D and E). In summary, the analysis revealed abnormalities in skin-wound healing in $\mathrm{AD}$-HIES patients that were characterized by delayed formation of granulation tissue, delayed reepithelialization, deficient neovascularization, and delayed restoration of dermal collagen by dermal fibroblasts. Deficiencies in these wound-healing processes may contribute to many of the AD-HIES pathologies, including inefficient repair of damaged soft tissues and structural abnormalities of connective tissues and arterial vessel walls.

Characterization of STAT3 signaling in human primary skin fibroblast culture model. In order to better understand the mechanisms underlying the nonimmune abnormalities of AD-HIES, we isolated fibroblasts from skin biopsies of healthy volunteers and AD-HIES patients (Supplemental Table 1) and analyzed the changes that AD-HIES mutations introduced into cell signaling networks.

We first confirmed intact STAT3 signaling and its responsiveness to IL- 6 and TNF- $\alpha$ in our skin fibroblast cell culture model (Supplemental Figure 2). TNF- $\alpha$ overproduction by AD-HIES patient peripheral blood mononuclear cells, neutrophils, and dendritic cells upon stimulation by LPS and impaired antiinflammatory STAT3-mediated IL-10 signaling in response to Staphylococcus aureus and peptidoglycan have been previously shown (29-31). Additionally, previous studies identified an important role for the interaction between TNF- $\alpha$ and STAT3 signaling during the initial steps of establishing a proper inflammatory and immune response following tissue injury (32). Therefore, in addition to basal conditions, we used TNF- $\alpha$ to mimic the local environment to which fibroblasts would be exposed in the damaged tissues. Our analysis showed that control fibroblasts expressed phosphorylated STAT3 under basal conditions and that the phosphorylation was increased by its direct activator IL- 6 and by TNF- $\alpha$ (Supplemental Figure 2A). The TNF- $\alpha$-induced STAT3 phosphorylation was prevented by the IL-6R antagonist, indicating that the effect is mediated by an autocrine signaling loop through TNF- $\alpha$-induced secretion of IL-6 (Supplemental Figure 2A). Indeed, measurement of IL- 6 in cell culture medium confirmed that both control and AD-HIES skin fibroblasts increased IL-6 secretion in response 
A

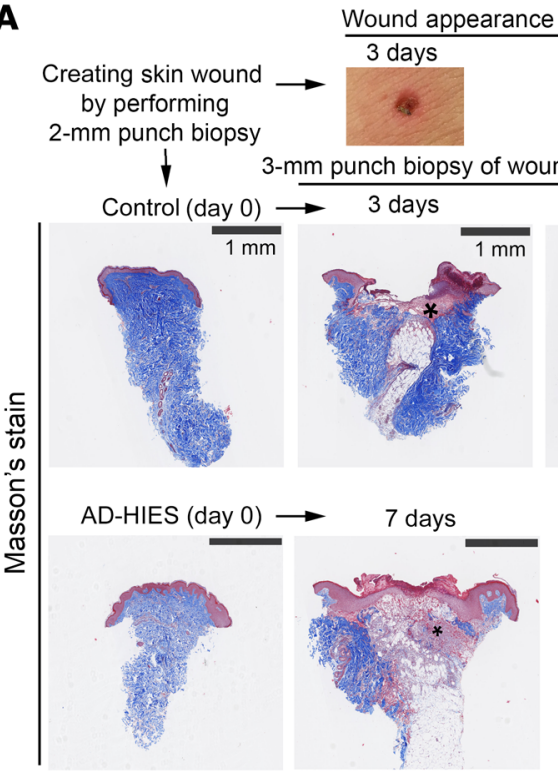

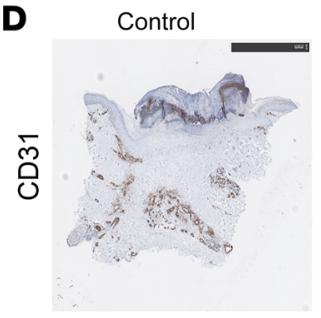
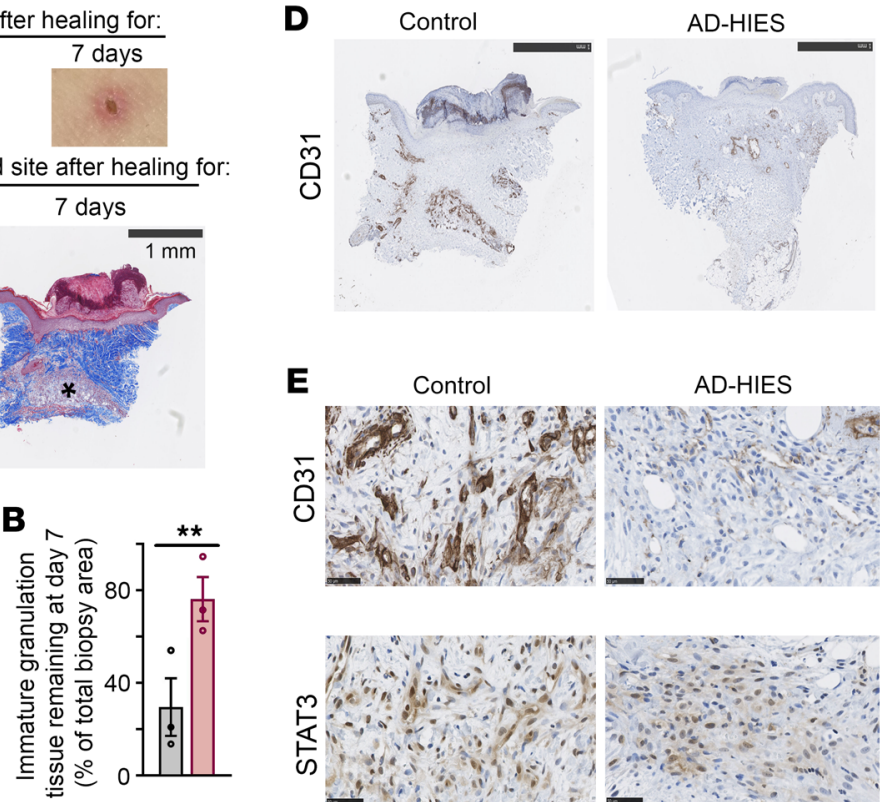

E
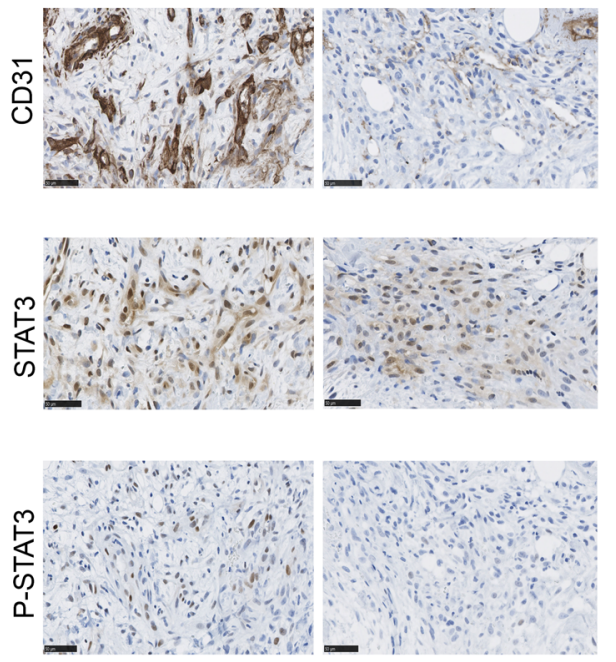
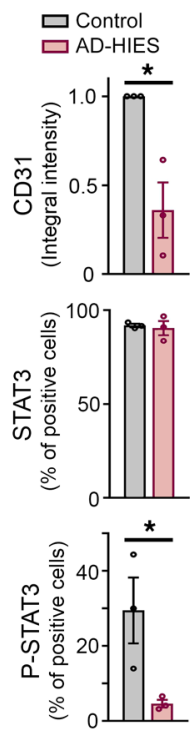

C

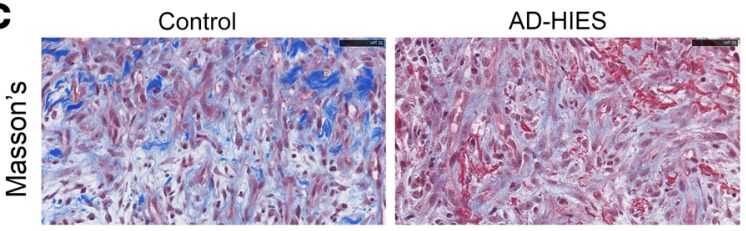

Figure 1. Delayed skin-wound healing in AD-HIES patients after tissue injury. (A) Overview of human cutaneous wound-healing model. A skin defect was created using a 2-mm-diameter skin punch biopsy tool ( $2 \mathrm{~mm}$ core biopsy of skin is excised to the depth of the deep dermis/dermal subcutaneous junction). Three or 7 days after the initial biopsy, a 3-mm skin punch biopsy of skin surrounding the original punch biopsy site was performed and the wound-healing process analyzed by Masson's trichrome and immunohistochemistry. Upper: representative images of wounds at days 3 and 7. Lower: representative images of perpendicularly bisected biopsies. Dark blue staining indicates abundant collagen. Red color indicates early granulation tissue (GT) with much less collagen (asterisks). (See also Supplemental Figure 1, A and B.) (B-E) Delayed GT formation, collagen restoration, and vascularization during skin-wound healing in AD-HIES patients. Three AD-HIES patients and 3 matched controls were analyzed. (B) At day 7, AD-HIES sections contain larger areas of less mature GT that lacks collagen. Data are plotted as percentages of biopsy section area occupied by GT with no or low collagen ( $n=3$ ). See also Supplemental Figure 1, C and D. (C) AD-HIES fibroblasts generate less collagen at day 7 after injury. Representative Masson's trichrome stain of GT (magnification of area labeled with asterisk in A). Scale bars: $50 \mu \mathrm{m}$. Collagen fibers show dark blue staining in the control, but weak to minimal staining in AD-HIES skin. (D) Decreased efficiency of new blood vessel formation in GT of AD-HIES. Representative images of staining for CD31 (a vascular endothelial cell marker) showed decreased vascularity and decreased endothelial cell staining in dermis. Scale bars: $1 \mathrm{~mm}$. (E) Impaired vascularization and STAT3 signaling in GT of AD-HIES patients. Left: representative images of staining for CD31, STAT3, and p-STAT3. Scale bars: $50 \mu \mathrm{m}$. Locations of the images are indicated by asterisks in A. Right: Quantification of the images (see Methods for details) $(n=3)$. Decreased CD31 content indicates deficient angiogenesis during wound healing. While almost all cells in GT express STAT3 protein both in control and in AD-HIES, percentages of cells that express p-STAT3 are greatly reduced in AD-HIES. Data are represented as mean \pm SEM. ${ }^{*} P<0.05 ;{ }^{* *} P<0.01$, 2-tailed unpaired $t$ test (B and E). See also Supplemental Figure 1. See Supplemental Table 1 for information about patient samples used.

to TNF- $\alpha$ (Supplemental Figure 2B). Following the verification of normal STAT3 signaling in the primary skin fibroblast culture model, we moved on to analyze the changes that AD-HIES mutations introduced in the STAT3 signaling networks.

RNA-Seq identifies a deficiency in cell signaling related to wound healing, angiogenesis, and ECM remodeling in AD-HIES skin fibroblasts. To study signaling pathways that could be affected by STAT3 mutations in AD-HIES during wound healing and to identify promising therapeutic targets, we performed RNA-Seq and subsequent gene set enrichment analysis (GSEA) (33) (Pathway Studio, Elsevier) and pathway-enrichment analysis (Figure 2 and Supplemental Figure 3) using primary skin fibroblasts isolated from skin biopsies of patients with AD-HIES as well as from normal control volun- teers (Supplemental Table 1). We compared global gene expression under basal conditions and after exposure to TNF- $\alpha$. RNA-Seq analysis identified many genes differentially expressed in AD-HIES fibroblasts relative to in the control, both in basal conditions and after treatment with TNF- $\alpha$ (Figure 2A). Many transcriptional changes were similar to the changes identified in previous analyses of patient leukocytes $(4,29,30,34,35)$, demonstrating overlaps in signaling pathways affected by AD-HIES STAT3 mutations in different cell types. Specifically, we found upregulation of many genes related to IFN signaling and host defense in skin fibroblasts from AD-HIES patients (Supplemental Table 2) and abnormal patterns of cytokine and chemokine expression (Supplemental Table 2). However, our analysis also identified additional pathways affect- 
A

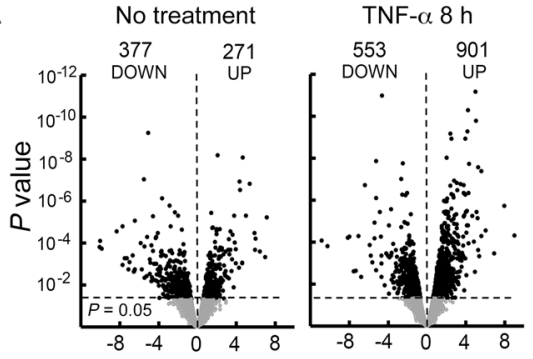

$\log _{2}$ (fold difference: AD-HIES vs. CT)

B

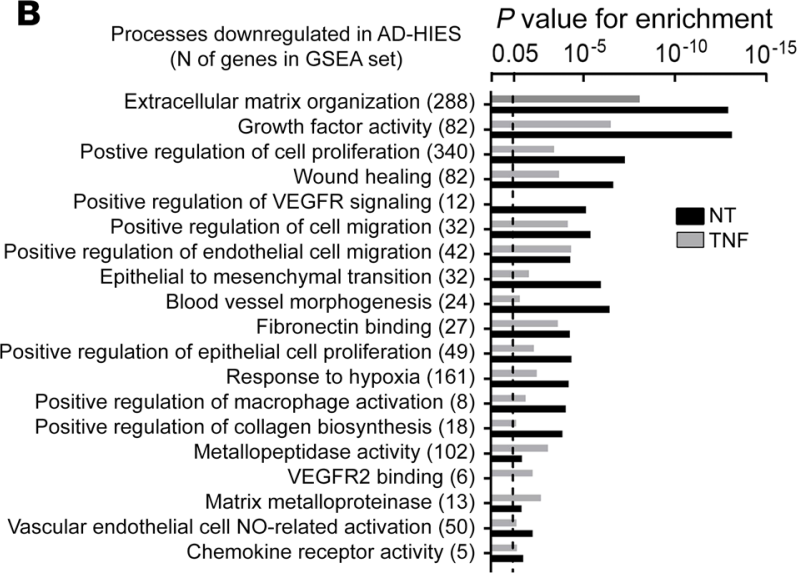

Figure 2. Deficiency in cell signaling related to wound healing, angiogenesis, and ECM remodeling in AD-HIES skin fibroblasts. Cultured skin fibroblasts generated from 3 AD-HIES patients and 3 normal volunteers were treated with or without TNF- $\alpha$ for 8 hours, and mRNA expression profiles were analyzed by RNA-Seq. (A) Overview of the differences in mRNA expression in AD-HIES and control (CT) skin fibroblasts. Expression data are plotted in the form of a volcano plot that plots fold difference versus $P$ value of the difference for each expressed gene $(n=3)$. (B-D) Biological processes and pathways related to wound healing, including tissue remodeling, angiogenesis, and growth factor signaling, are downregulated in AD-HIES skin fibroblasts. (B) Biological processes that were identified by GSEA as significantly downregulated in AD-HIES skin fibroblasts. For each gene set, name of biological process is shown on the left with number of genes included in the set. Bars on the right plot $P$ values for the gene set enrichment representing the degree of confidence for concordant decrease of expression of the genes in the set. Analysis was performed with Pathway Studio software. (C) Top: enrichment plot for the wound-healing gene signature (Gene Ontology [GO] 0042060) in AD-HIES compared with WT. Bottom: visualization of the expression of the signature genes. (D). Top: enrichment plot for the positive regulation of angiogenesis gene signature (GO 0045766) in AD-HIES compared with control. Bottom: visualization of the expression of the signature genes. CSEA for $\mathbf{C}$ and $\mathbf{D}$ was performed with Broad Institute software. See also Supplemental Figure 3 and Supplemental Table 2. See Supplemental Table 1 for information about patient samples used in these experiments.

ed in AD-HIES skin fibroblasts that were consistent with AD-HIES nonimmune pathologies and seemed a promising target to investigate for a clinically impactful therapy for these pathologies. Thus, GSEA showed that multiple pathways related to wound healing, tissue development, and tissue remodeling were downregulated in AD-HIES skin fibroblasts, both in basal conditions and in the presence of TNF- $\alpha$ (Figure 2B). Targeted GSEA confirmed concordant decreased expression of genes related to both wound healing (Figure 2C) and the positive regulation of angiogenesis (Figure 2D). Further pathway analysis performed on genes that were differentially expressed in AD-HIES identified the ECM-remodeling pathway as the most affected (Supplemental Figure 3 and Supplemental Table 2). Decreased expression of several MMPs, ECM proteins, and growth factors that participate in ECM metabolism, tissue remodeling, and angiogenesis during wound healing (Supplemental Table 2 and refs. $36-38$ ) is consistent with nonimmunological port with ECM proteins and proangiogenic factors released by immune cells and fibroblasts as well as by intrinsic deficiencies in the angiogenic responses of endothelial cells, which are themselves elicited by AD-HIES STAT3 mutations. Therefore, we next performed RNA-Seq of pathways dysregulated in HUVECs isolated from umbilical cords of healthy babies and babies born with AD-HIES STAT3 dominant negative mutations (Supplemental Figure 4). GSEA demonstrated that, as in skin fibroblasts (Supplemental Table 2) and immune cells (3, 4), AD-HIES HUVECs have increased expression of genes related to IFN signaling (Supplemental Figure 4, C and D). However, unlike fibroblasts, HUVECs did not show deficiencies in pathways related to wound healing and angiogenesis and even demonstrated significant upregulation of these pathways (Supplemental Figure 4, B and C). Consistently, AD-HIES HUVECs performed similarly to control HUVECs in an endothelial cell tube formation assay performed in medium 
fully supplemented with proangiogenic growth factors, suggesting preserved intrinsic angiogenic potential (Supplemental Figure $4 \mathrm{E}$ ). The responses identified in these experiments analyzing HUVECs with AD-HIES STAT3 mutations are similar to previous findings from endothelial-specific Stat3-knockout mice showing exaggerated inflammatory response and normal performance in an endothelial cell tube formation assay (44). Therefore, we proceeded with more detailed studies of deficiencies that AD-HIES fibroblasts display in paracrine support of ECM remodeling and angiogenesis during repair of tissue damages.

Confirmation of STAT3 dependence of changes detected by RNASeq in AD-HIES skin fibroblasts. By targeted quantitative PCR (qPCR), we confirmed reduced expression of $M M P 1, M M P 3, M M P 9$, and VEGFR2 mRNAs in AD-HIES fibroblasts (Supplemental Figure $5 \mathrm{~A})$. We also confirmed that this deficiency is STAT3 dependent; it could be rescued by expressing WT STAT3 in the AD-HIES fibroblasts (Supplemental Figure 5B) and reproduced by shRNA-mediated knockdown of STAT3 in control fibroblasts (Supplemental Figure 5C). MMP1, MMP3, and MMP9 genes are direct targets of STAT3 (45). ChIP assay demonstrated that the binding efficiency of STAT3 to promoters of these genes is diminished in AD-HIES fibroblasts (Supplemental Figure 5D), confirming that their reduced expression in AD-HIES fibroblasts is STAT3 dependent.

Decreased ability of AD-HIES skin fibroblasts to support angiogenesis in cell culture models. Following the identification via RNA-Seq of a global decrease in the gene expression of processes related to tissue remodeling and angiogenesis, we aimed to verify these findings functionally. We demonstrated, via RNA-Seq and qPCR, decreased mRNA expression of several MMPs (Supplemental Figure 3, Supplemental Figure 5A, and Supplemental Table 2) and major angiogenesis-related growth factors (FGF-2, PLGF, and VEGFA) in AD-HIES skin fibroblasts (Supplemental Table 2 and Figure $3 \mathrm{~A}$ ). This corresponded to decreased secretion of these MMPs and several growth factors by the fibroblasts into surrounding cell culture media (Figure 3B). Correspondingly, cell culture medium collected from $\mathrm{AD}-\mathrm{HIES}$ fibroblasts had a reduced ability to support angiogenesis in the endothelial cell tube formation assay (Figure 3C).

Decreased ability of AD-HIES skin fibroblasts to support angiogenesis in iPSC-derived teratoma model. We next tested the proangiogenic potential of AD-HIES fibroblasts by studying their ability to promote the growth of teratomas derived from iPSCs after subcutaneous injection into NOD/SCID- $\gamma$ (NSG) immunodeficient mice (46). For these experiments, we used iPSCs generated from our control and AD-HIES skin fibroblast cell lines (47). Surprisingly, compared with teratomas derived from control iPSCs, teratomas derived from AD-HIES iPSCs were small and avascular, suggesting a deficiency in blood vessel formation, thus providing us a model for investigating angiogenic capacities of human cells (fibroblasts) transplanted into human tissue (teratoma) (Figure 3D and Supplemental Figure 6A). Despite being very small, AD-HIES iPSC-derived teratomas contained structures representing all 3 lineages, indicating similar differentiation potential (Supplemental Figure 6B). Indeed, mixing of AD-HIES iPSCs with normal skin fibroblasts resulted in restoration of the teratoma growth rate (Figure 3D and Supplemental Figure 6C), accompanied by more efficient blood vessel formation, evidenced from increased proportion of $\mathrm{CD}^{+} 1^{+}$cells (Figure 3D and Supplemental Figure 7).
However, AD-HIES skin fibroblasts failed to provide such support for teratoma growth (Figure 3D, Supplemental Figure 6C, and Supplemental Figure 7), consistent with the decreased ability of AD-HIES fibroblasts to secrete proangiogenic factors.

Decreased ability of AD-HIES skin fibroblasts to support angiogenesis in mouse hind-limb ischemia model. To further investigate the ability of AD-HIES fibroblasts to support angiogenesis in the setting of tissue damage and remodeling, we used the murine model of hind-limb ischemia (HLI). In this model, surgical ligation of the femoral artery stops blood supply to the hind limb and stimulates angiogenesis in the calf muscles, leading to increased capillary density $(48,49)$. We tested the ability of AD-HIES fibroblasts to facilitate recapillarization after femoral artery ligation in NSG mice. At baseline, NSG immunodeficient mice have decreased recapillarization ability and require additional support with proangiogenic factors to be able to restore blood perfusion after femoral artery ligation (50). Injection of control fibroblasts into the calf muscle after femoral artery ligation stimulated angiogenesis, leading to restoration of blood perfusion (Figure 3, E and F) due to increased capillary density (Figure 3G). However, injection of AD-HIES skin fibroblasts failed to increase capillary density (Figure 3G) and did not restore blood perfusion (Figure 3, E and F). These results indicate that AD-HIES fibroblasts were not able to provide proangiogenic factors that were missing in immunodeficient mice due to lack of normal immune response initiated by injury, while normal skin fibroblasts were able to compensate for this deficiency by secreting necessary proangiogenic factors (Figure 3, E-G).

$H I F-1 \alpha$-signaling deficiency in AD-HIES fibroblasts predicted by RNA-Seq. After confirmation of the RNA-Seq-predicted angiogenesis deficiency at a functional level, we sought to identify specific STAT3-dependent signaling targets that mediate such dysregulation. Among the biological processes that we identified by GSEA as significantly downregulated in $\mathrm{AD}$-HIES skin fibroblasts was response to hypoxia (Figure $2 \mathrm{~B}$ ). HIF-1 $\alpha$, a master regulator of the hypoxic response (51-53), is also implicated in the regulation of a wide range of conditions characterized by ischemia and inflammation, including wound healing and tissue remodeling $(54,55)$. Indeed, GSEA showed a concordant decrease in mRNA expression of HIF-1 $\alpha$ transcriptional targets in AD-HIES fibroblasts both in basal conditions and after stimulation with TNF- $\alpha$ (Supplemental Figure 8A, Figure 4A, and Supplemental Table 2), indicative of a STAT3-dependent decrease of HIF- $1 \alpha$ transcriptional activity in AD-HIES skin fibroblasts. Indeed, Western blot analysis showed that the HIF-1 $\alpha$ protein level was increased in response to TNF- $\alpha$ in control but not in AD-HIES fibroblasts (Figure 4B), paralleled by increased transcriptional activity in control fibroblasts but not in AD-HIES fibroblasts (Supplemental Figure 8B). At the same time, response to hypoxia was largely preserved: the HIF-1 $\alpha$ protein level in the AD-HIES fibroblasts increased in response to hypoxia to the same degree as that of the control fibroblasts (Figure 4C) and transcriptional activity was also increased, but to slightly lower levels than in control fibroblasts (Supplemental Figure 8B).

STAT3-dependent decrease in HIF-1 $\alpha$ transcription in AD-HIES skin fibroblasts. We explored 2 possible mechanisms for the decreased expression level of HIF- $1 \alpha$ protein in AD-HIES: decreased transcription and increased rate of degradation. Such mechanisms and possible STAT3 involvement have been demon- 

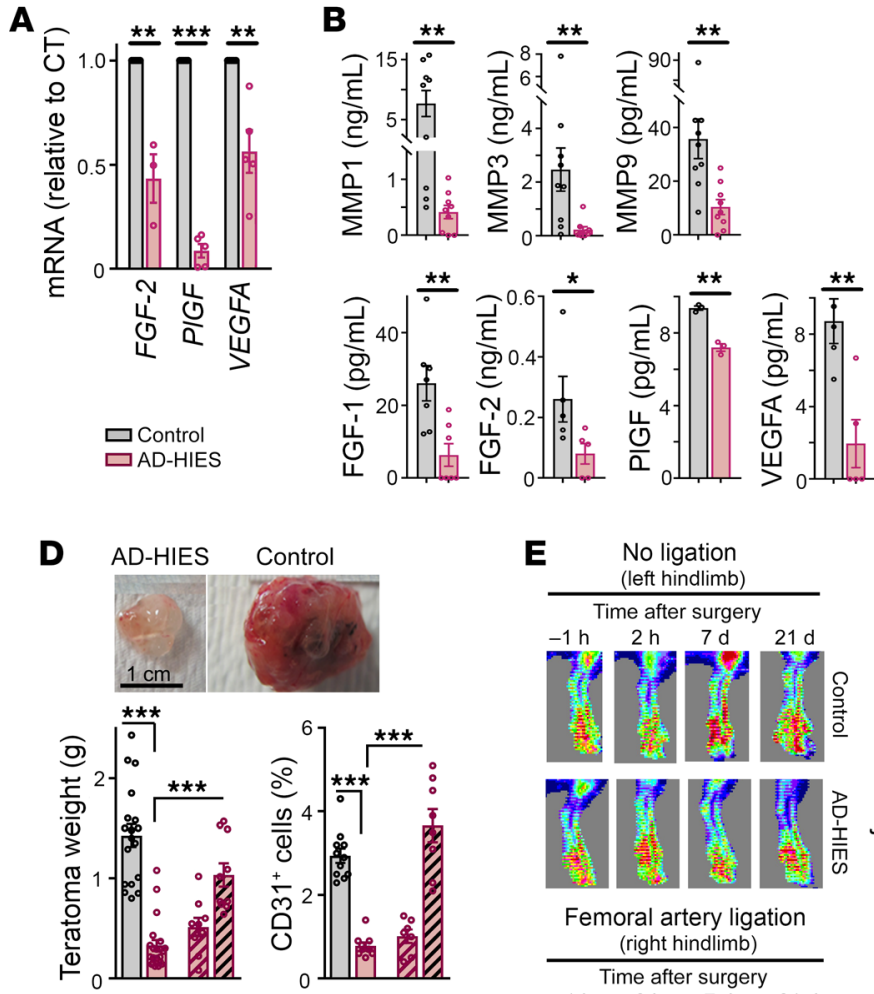

Cells injected into hindlimbs of NSG mice: $\square$ Control iPSC $\square$ AD-HIES IPSC

AD-HIES iPSC + a AD-HIES fibro ad Control fibro
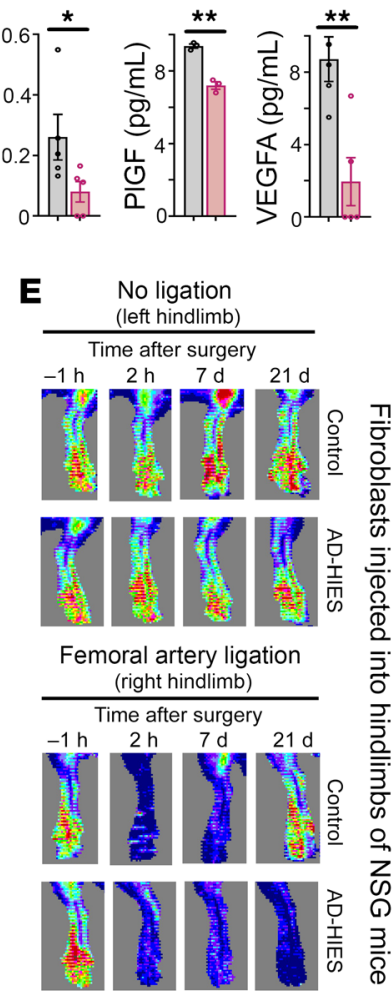

C
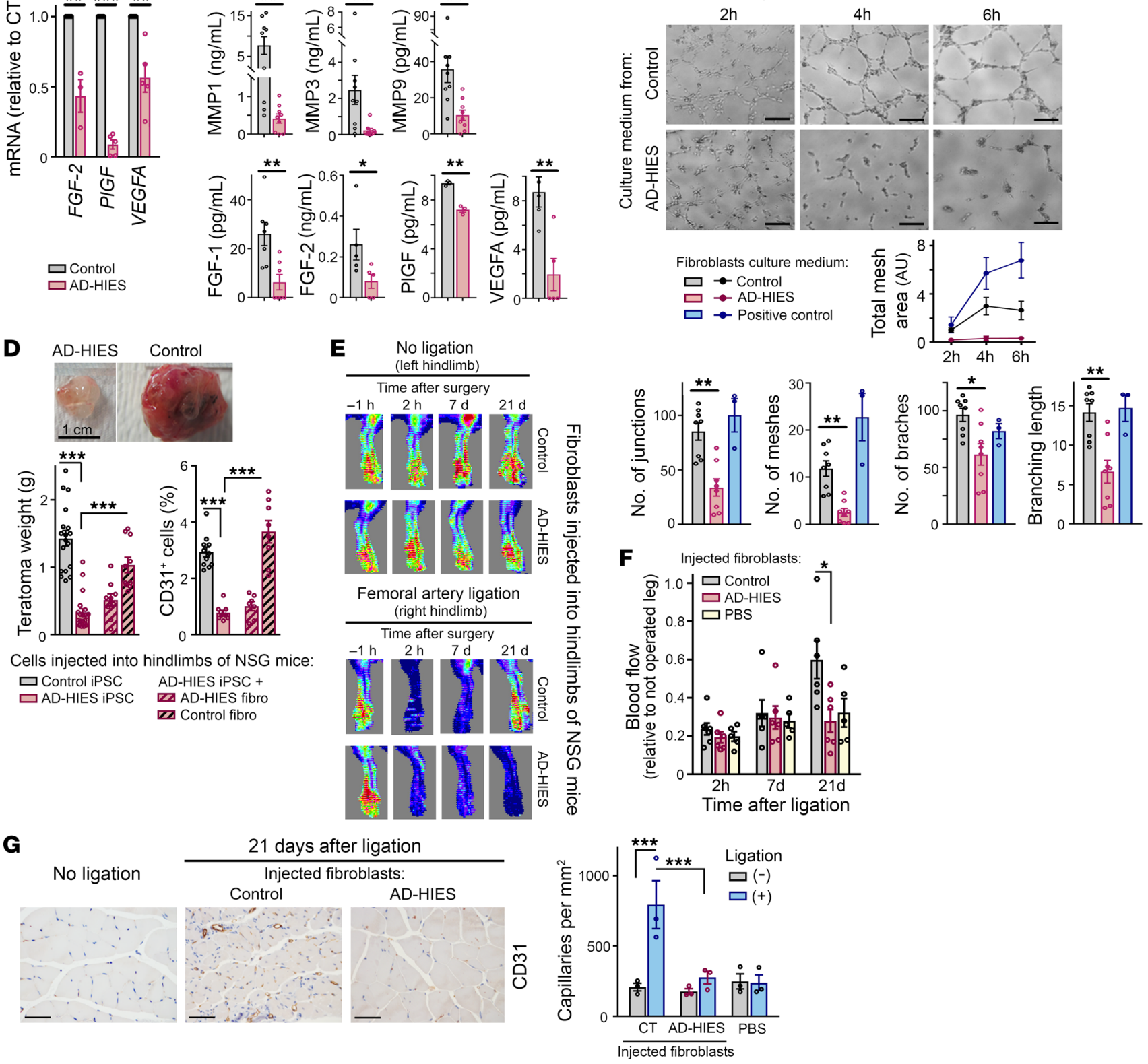

Figure 3. Decreased ability of AD-HIES skin fibroblasts to support angiogenesis in cell culture and mouse models. (A) AD-HIES skin fibroblasts (SFs) have decreased mRNA expression of angiogenic factors $(n=5)$. (B) AD-HIES skin fibroblasts secrete fewer angiogenic factors $(n=3-9)$. (C) Cell culture medium collected from AD-HIES fibroblasts has reduced ability to support angiogenesis in endothelial cell tube formation assay. HUVECs were cultured in culture medium collected from control and AD-HIES SFs. Left: representative images. Scale bars: $100 \mu \mathrm{m}$. Right: quantification of tubule structures ( $n=8$ ). (D) AD-HIES SFs fail to promote growth and vessel formation of AD-HIES iPSC-derived teratomas. iPSCs generated from 3 control and 3 AD-HIES SFs lines were injected alone or as mixture with SFs into hind limbs of NSG mice. In 8 weeks, teratoma weights $(n=10-18)$ and percentages of CD31+ cells $(n=7-11)$ were analyzed. See also Supplemental Figures 6 and 7. (E-C) AD-HIES fibroblasts fail to support restoration of blood perfusion after femoral artery ligation in NSC immunodeficient mice. HLI was induced by ligation of the femoral artery. Control, AD-HIES fibroblasts, or PBS was injected into calf muscle of ligated hind limb 1 day after surgery. (E and F) Estimation of blood flow by laser Doppler imaging. (E) Representative Laser Doppler images. (F) Quantification $(n=5-6)$. (C) Estimation of capillary density. Left: representative images of immunostaining with anti-CD31. Scale bars: $50 \mu \mathrm{m}$. Right: quantification $(n=3)$. Blood flow and capillary density are increased after injection of control fibroblasts but not after injection of AD-HIES fibroblasts or PBS. Data are represented as mean \pm SEM. ${ }^{*} P<0.05 ;{ }^{* *} P<0.01 ;{ }^{* *} P<0.001$, Mann-Whitney rank sum test (A); 2-tailed unpaired $t$ test (B); 1 -way ANOVA followed by Holm-Šidák multiple comparisons test (C, D, and $\mathbf{C})$; Kruskal-Wallis test followed by Dunn's multiple comparisons test (F). See Supplemental Table 1 for information about patient samples used.

strated in a number of previous studies (56-58). The promoter of the HIF1 $\alpha$ gene contains several STAT3-binding sites (Supplemental Figure 9) and binding of STAT3 to the HIF1 $\alpha$ promoter has been demonstrated both in cancer cells (57) and mouse T cells (56). Our analysis showed that STAT3 binds to the HIF1 $\alpha$ promoter in normal skin fibroblasts, but that binding is impaired in AD-HIES (Figure 4D). Consistently, a smaller amount of HIF-1 $\alpha$ mRNA is present in AD-HIES fibroblasts (Figure 4E). Analysis of HIF-2 $\alpha$ mRNA 
expression, the functions of which substantially overlap with those of HIF- $1 \alpha$ (59), demonstrated that HIF- $2 \alpha$ was expressed at lower levels compared with HIF- $1 \alpha$ mRNA, was not differently expressed in control and AD-HIES cells, and was not affected by TNF- $\alpha$ (Figure $4 \mathrm{E}$ ), suggesting that it is less likely to be a main contributor to AD-HIES phenotypes. To confirm the STAT3 dependence of reduced expression of HIF-1 $\alpha$, we performed knockdown and overexpression experiments. Consistent with the STAT3 dependency, siRNA-mediated knockdown of STAT3 in control fibroblasts decreased HIF-1 $\alpha$ mRNA and protein levels (Supplemental Figure 10, A-C), while expression of WT STAT3 in the AD-HIES fibroblasts elevated HIF-1 $\alpha$ mRNA and protein levels (Supplemental Figure 10, D-F). In addition, HIF-1 $\alpha$ mRNA knockdown in control fibroblasts decreases MMPs and KDR mRNA (Supplemental Figure 8C). Similar results were seen with mRNA knockdown of STAT3 (Supplemental Figure 5C). Taken together, these results indicate that both STAT3 and HIF-1 $\alpha$ regulate transcription of relevant genes. These results demonstrate that STAT3-dependent deficiency in the regulation of HIF- $1 \alpha$ transcription in AD-HIES is a contributing mechanism to the decrease in target genes (Figure 4A, Supplemental Figure 8A, and Supplemental Table 2).

STAT3-dependent decrease in HIF-1 $\alpha$ protein stability in AD-HIES skin fibroblasts. Another possible mechanism for increase of HIF-1 $\alpha$ protein level is one that operates in response to hypoxia through its stabilization due to decreased activity of prolyl hydroxylases (PHDs) that hydroxylate HIF-1 $\alpha$ protein and target it for von-Hippel Lindau-dependent (VHL-dependent) ubiquitination and degradation (52). Therefore, in the next series of experiments, we determined whether AD-HIES mutations affected HIF- $1 \alpha$ protein stability. Our measurements of HIF- $1 \alpha$ protein degradation rates demonstrated that TNF- $\alpha$ stabilizes HIF- $1 \alpha$ protein in normoxic conditions in control fibroblasts, but fails to do so in AD-HIES fibroblasts (Supplemental Figure 8D and Figure 4, F-H). Similar deficiency in stabilization of HIF- $1 \alpha$ protein in response to TNF- $\alpha$ occurs when the level of STAT3 is decreased by siRNA in control fibroblasts, confirming that stabilization is STAT3 dependent (Supplemental Figure 10G). These results indicate that HIF- $1 \alpha$ destabilization in AD-HIES contributes to deficiencies in its signaling network in response to TNF- $\alpha$. Mechanistic analysis demonstrated that deficient HIF-1 $\alpha$ signaling in AD-HIES skin fibroblasts is caused by dysregulation of its expression on both transcriptional and protein stability levels.

Decreased HIF-1 $\alpha$ expression during skin-wound healing and in coronary arteries of AD-HIES patients. Having found deficient HIF- $1 \alpha$ signaling in cultured AD-HIES skin fibroblasts, we aimed to verify whether these findings can be confirmed in AD-HIES patients in vivo. Immunohistochemical analysis demonstrated decreased HIF- $1 \alpha$ protein expression in granulation tissue of AD-HIES skin wounds (Figure 5A) and in coronary arteries from AD-HIES patients (Figure 5B). Consistent with RNA-Seq and qPCR results in fibroblasts, we found decreased expression of $M M P 1$ and $M M P 3$ mRNAs in AD-HIES coronary arteries (Figure $5 \mathrm{C}$ and Supplemental Figure 11B).

Decreased blood perfusion by the vasa vasorum in the coronary artery walls of AD-HIES patients. To find out whether vascular structural abnormalities could be promoted by deficient angiogenesis, we analyzed the angiogenic response and perfusion of
AD-HIES coronary arteries through the analysis of the vasa vasorum, a vascular network located in the adventitia that supplies oxygen and nutrients to larger arteries (diameter $>0.5 \mathrm{~mm}$ ). Both the formation of the vasa vasorum during normal arterial growth and expansion of the vasa vasorum during pathological arterial wall remodeling are driven by local secretion of proangiogenic factors by medial smooth muscle cells (SMCs) and adventitial fibroblasts in response to hypoxia and inflammation (60). Vasa vasorum density as well as overall blood perfusion was reduced in AD-HIES coronary arteries as compared with control coronary arteries (Figure 5D), consistent with deficient angiogenesis.

Altered ECM composition in the coronary artery walls of AD-HIES patients. Arterial tortuosity, dilatation, and aneurysms in patients with AD-HIES are not accompanied by inflammation or calcification. In addition, the response to atherosclerosis is abnormal (13, $14,61)$, suggesting intrinsic ECM abnormalities as a likely etiology. Unlike in the skin-wound-healing process, in which fibroblasts play a major role in ECM remodeling, arterial wall ECM's master regulators are SMCs. We hypothesized that the dysregulation of ECM remodeling and angiogenesis-related gene networks seen in AD-HIES fibroblasts (Figure 2 and Supplemental Figure 3) could be similar in SMCs and translate to altered ECM composition and mechanical properties of the arterial walls in AD-HIES patients. Indeed, immunohistochemical analysis of coronary arteries from AD-HIES patients showed an abnormal composition of major ECM components in the arterial wall (Figure 5, E and F, Supplemental Figure 11A, and ref. 62). Specifically, AD-HIES coronary arteries contained more collagen I and elastin in the adventitia, more collagen I and collagen IV in the intima, and fewer laminins in all layers of the arterial wall (Figure 5, E and F). Correlation analysis revealed strong associations between the abundance of elastin and the perfusion of the coronary artery wall $(R=-0.82$, $P=0.003)$, with clear separation of control and AD-HIES coronary artery (CA) walls on the correlation curve (Supplemental Figure 11C), suggesting existence of coordinated interdependent regulation of these structural components of the vessel walls in a STAT3-dependent manner. In summation, the changes in ECM composition and blood perfusion of the arterial walls mediated by STAT3-dependent signaling in medial SMCs and adventitial fibroblasts could be an underlying cause of the arterial tortuosity and aneurysms observed clinically in $\mathrm{AD}$-HIES patients.

Pharmacological stabilization of HIF-1 $\alpha$ restores ability of AD-HIES skin fibroblasts to support angiogenesis in cell culture. Verification of decreased HIF-1 $\alpha$ protein expression in AD-HIES patients in vivo during wound healing (Figure 5A) and in their coronary arteries (Figure 5B) suggested that pharmacological stabilization of HIF- $1 \alpha$ could correct AD-HIES-related abnormalities. The degradation of HIF-1 $\alpha$ protein, which occurs under normoxic conditions, is mediated by PHDs $(63,64)$. We used 2 inhibitors of PHDs, dimethyl fumarate (DMF) and daprodustat $(26,55)$, to stabilize expression of HIF- $1 \alpha$ protein and to test the effect of HIF- $1 \alpha$ signaling restoration on angiogenesis in AD-HIES. Treatment of AD-HIES fibroblasts with both daprodustat and DMF stabilized HIF-1 $\alpha$ protein under normal cell culture conditions and hypoxia as well as in the presence of TNF- $\alpha$ (Figure 6, A and B). Stabilization of HIF- $1 \alpha$ by DMF increased its transcriptional activity in a hypoxia response element (HRE) luciferase assay (Figure 6C). 
A
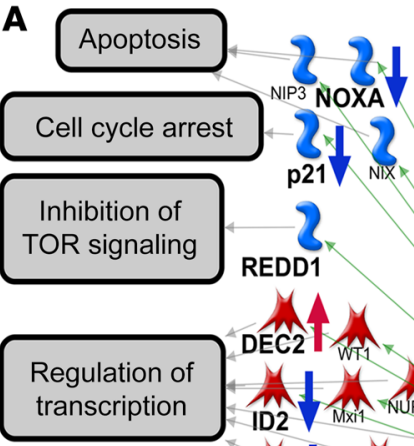

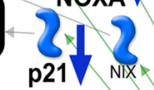
2 REDD1

x. DEC2 PAN transcription

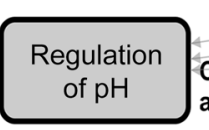
ID2 2 Axi1 NURT A 1 RORRapha CITED2 $)($ 14 Carbonic
anhydrase XII Carbonic
anhydrase Transferrin Eerruloplasmin

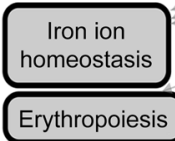
metabolism
Lipid

\section{$5^{5} 21$}

Adipophilin

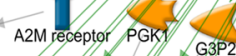
A2M recentor PGKA GSP2

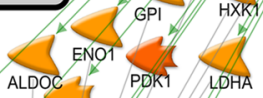

$Y^{2} Y^{\text {PFKL }}$ 2ै। GLUT1 F263 ALDOA LOXL4 oxidase

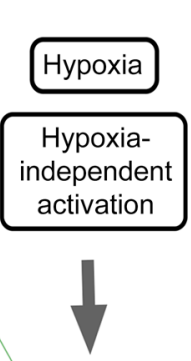

HIF-1A
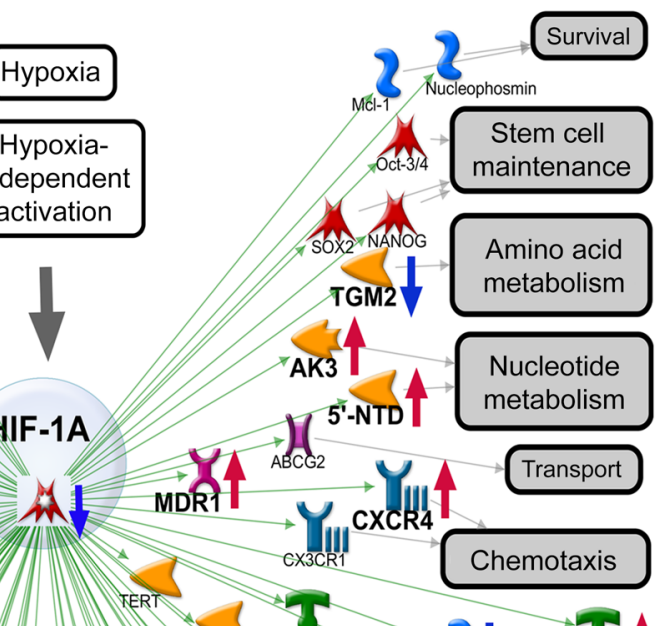
减

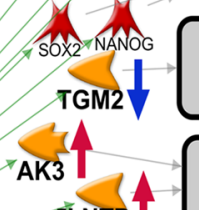

Amino acid metabolism $(5$ $5^{5}-\mathrm{NTD}$

Nucleotide metabolism

Transport

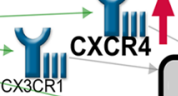

Chemotaxis
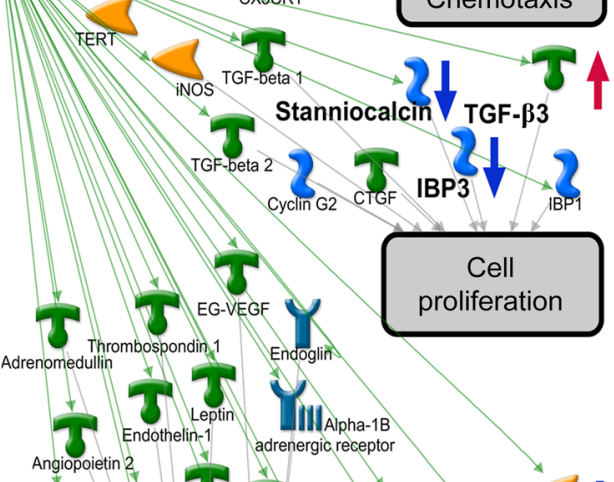

$y+5 t$ HGF ${ }^{\text {PDGF-B }}$ PAI1 VEGF-A Oxygenase 1 GLUT3 PKM2 HXK2 I MMP-9 LOXL2 P4HA2

receptor
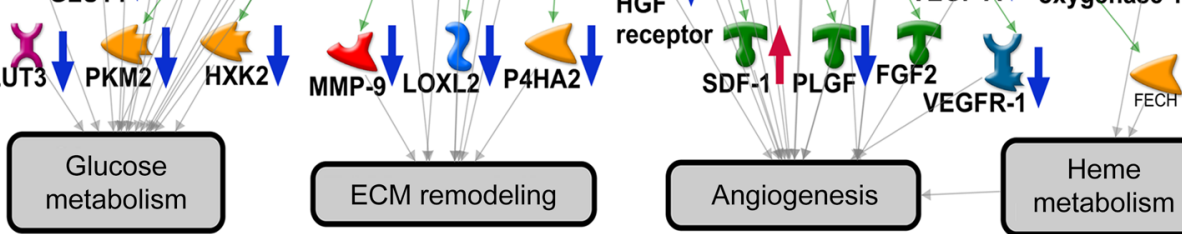

Angiogenesis

Heme

ECM remodeling

3 Receptor ligand

AD-HIES vs CONTROL

3 Enzyme

2 Binding prptein

$\downarrow$ DOWN † UP

Metalloprotease

E HIF-1 $\alpha$ HIF-2 $\alpha$

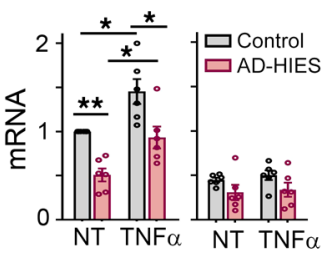

$\mathbf{F}$

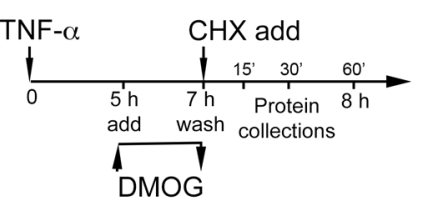

G No treatment กิ $\sum_{0}^{O} \frac{\mathrm{CHX}, \min }{5153060}$

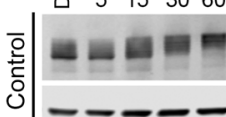
$---$

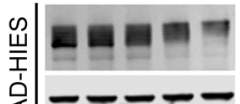

定 --
Mranscription factor

Xransporter

Y Receptor
B
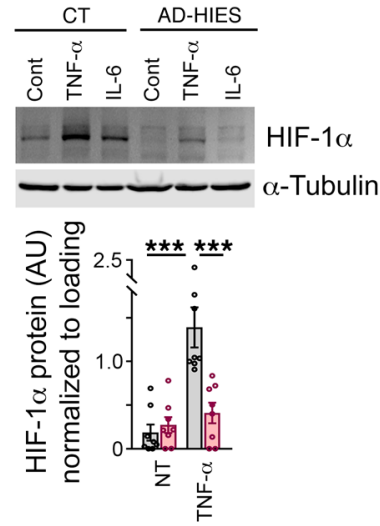

C $\mathrm{CT}$ AD-HIES $\square$ Control z $\stackrel{\circ}{\circ} \stackrel{\circ}{\circ}$

$-\quad-$ HIF-1 $1 \alpha$ $-\alpha$-Tubulin

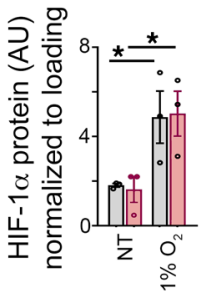

D

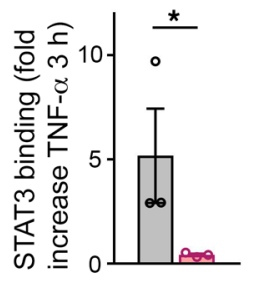

HIF1 $\alpha$ promoter (STAT3 binding site)
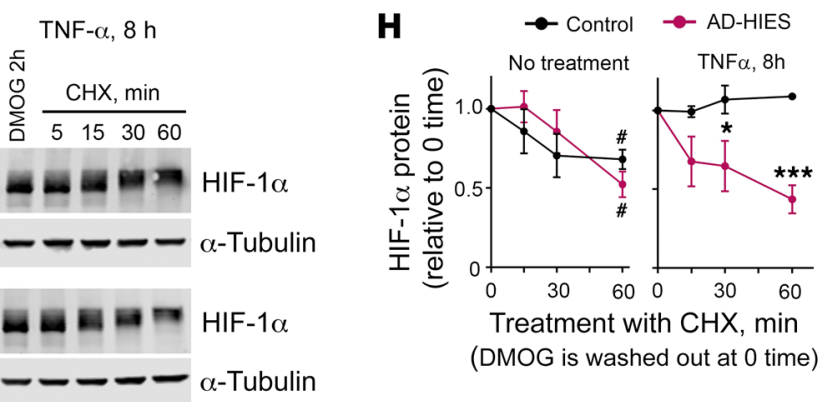
Figure 4. Deficient HIF-1 $\alpha$ signaling in AD-HIES SFs at transcriptional and protein stabilization levels. (A) RNA-Seq pathway map showing decreased expression of many HIF-1 $\alpha$ transcriptional targets in AD-HIES fibroblasts ( $P=5.3 \mathrm{E}-12$; FDR $=4.2 \mathrm{E}-9$; GeneGo Metacore software). See Supplemental Table 2 for expression changes. (B) AD-HIES fibroblasts are deficient in ability to stabilize HIF-1 $\alpha$ protein in response to cytokines. Cells were treated with TNF- $\alpha$ and IL- 6 for 8 hours. Representative Western blot (upper panel) and quantification by densitometry (lower panel) are shown $(n=8)$. (C) AD-HIES SFs stabilize HIF-1 $\alpha$ under hypoxic conditions $\left(1 \% \mathrm{O}_{2}\right.$ for 8 hours). Representative Western blot and quantification $(n=3)$. (D) TNF- $\alpha$ increases STAT3 binding to promoter of HIF1 $\alpha$ gene in control SFs but not in AD-HIES (ChIP; see Supplemental Figure 9 and Supplemental Table 3). Data are plotted as fold increase of STAT3 binding after treatment with TNF- $\alpha$ (see Methods) $(n=3)$. (E) HIF-1 $\alpha$ mRNA levels were decreased in AD-HIES fibroblasts. $(n=6)$. (F-H) TNF- $\alpha$ decreased degradation rate of HIF-1 $\alpha$ in control fibroblasts, but not in AD-HIES, indicating STAT3-dependent stabilization of HIF-1 $\alpha$ and that this stabilization is STAT3 dependent. To measure rate of HIF-1 $\alpha$ protein degradation, de novo protein synthesis was inhibited with cycloheximide (CHX). (F) Experimental design for estimation of HIF-1 $\alpha$ stability. To increase level of HIF-1 $\alpha$, cells were treated with PHD inhibitor dimethyloxalylglycine (DMOC) that was washed out before addition of CHX. (C) Representative Western blot images showing dynamics of HIF-1 $\alpha$ decrease after addition of CHX. (H) Quantification of Western blots ( $n=3$; asterisks, relative to control; pound signs, relative to 0 time). See also Supplemental Figure 8D. Data are represented as mean \pm SEM. ${ }^{*, \#} P<0.05$; ${ }^{* *} P<0.01$; ${ }^{* *} P<0.001$, 2-tailed unpaired $t$ test (B, D, E, and $\mathbf{H})$; 1-way ANOVA followed by Holm-Šidák multiple comparisons test (C). See Supplemental Table 1 for information about patient samples used.

Both DMF and daprodustat were able to increase mRNA levels (Figure 6D) and secretion of proangiogenic factors (VEGF-A and FGF-2) in cell culture medium (Figure 6E). Consistently, cell culture media collected from AD-HIES fibroblasts after treatment with DMF acquired an ability that it otherwise lacked (Figure 3C) to support angiogenesis in an endothelial cell tube formation assay (Figure 6F). Increased VEGFA secretion by AD-HIES fibroblasts was a major contributor to such improvement, given that the neutralization of VEGFA by addition of the anti-VEGF monoclonal antibody bevacizumab prevented the beneficial effect of the DMF treatment (Figure 6F).

Vascular structural abnormalities and deficient angiogenesis and wound healing in an AD-HIES mouse model. Having found that HIF$1 \alpha$ stabilization is able to improve the proangiogenic properties of AD-HIES human skin fibroblasts in culture, we then tested the ability of HIF-1 $\alpha$ stabilizers to correct angiogenesis and wound-healing deficiencies in the AD-HIES mouse model. The mouse model of AD-HIES that expresses a dominant negative mutation in STAT3 recapitulates multiple aspects of AD-HIES immunological features, including elevated serum IgE and the failure to generate Th17 cells (65). Nonimmunological features of AD-HIES have not been accessed in this model before. Casting of the coronary arterial network in the AD-HIES mice identified structural abnormalities in the coronary arteries that resemble vascular abnormalities seen in AD-HIES patients, such as increased tortuosity and abnormal branching (Figure 7, A-C, Supplemental Figure 12, A and B, and Supplemental Videos 1 and 2). Consistent with the AD-HIES angiogenesis defects identified in human fibroblasts, these mice have a diminished ability to activate recapillarization and restore blood perfusion after ligation of the femoral artery when compared with WT mice (Figure 7, D and E). Similarly to deficient skin- wound healing in AD-HIES patients (Figure 1 and Supplemental Figure 1), healing in the mouse model was also delayed (Figure 7, $\mathrm{H}$ and $\mathrm{I}$, and Supplemental Figure 12, C-E).

$H I F-1 \alpha$ stabilizers improve angiogenesis and wound healing in an AD-HIES mouse model. We then tested the ability of HIF-1 $\alpha$ stabilizers to improve angiogenesis-related deficiencies in AD-HIES mice. Treatment by daily gavage with DMF improved recapillarization after ligation of the femoral artery in the AD-HIES mice (Figure 7F) and restored growth of teratomas from AD-HIESderived iPSCs in NSG mice (Figure 7G). To study skin-wound healing in mice, we used a splinted wound-healing model (66, 67). Wound splinting minimizes skin contraction (a main mode of wound healing in mice) and stimulates healing through epithelialization and granulation tissue formation, which resembles the human skin-wound-healing process (Figure $7 \mathrm{H}$ and Supplemental Figure 12, C-E). We used 2 HIF-1 $\alpha$ stabilizers, DMF and daprodustat, to decrease the possibility that off-target drugs effects would be the main contributors for observed changes. Topical treatment of wounds with both DMF and daprodustat accelerated wound closure in AD-HIES mice (Figure 7, $\mathrm{H}^{-} \mathrm{J}$ ). Immunohistochemical analysis demonstrated tissue-wide phosphorylation of Stat 3 in the wound area, but not in undamaged skin (Figure 7K), verifying the importance of Stat3-dependent signaling in the wound-healing process. Effectiveness of topical treatment with DMF for increasing the HIF-1 $\alpha$ level in granulation tissue was verified by immunostaining (Figure 7, L and $\mathrm{M}$ ).

HIF-1 $\alpha$ stabilizers improve TH17 differentiation. The ability of HIF- $1 \alpha$ stabilization to normalize the STAT3 dominant negative phenotype was not limited to angiogenesis. DMF also improved the differentiation of naive STAT3LOF CD $4^{+} \mathrm{T}$ cells isolated from AD-HIES patients into Th17 cells, a process that is affected by HIF- $1 \alpha$ activity (56) and is otherwise highly impaired in AD-HIES, potentially underlying AD-HIES patients' susceptibility to Candida and staphylococcal infections (ref. 68 and Supplemental Figure 13).

\section{Discussion}

Rare diseases, especially ones with an identified genetic cause, provide unique opportunities for understanding the biological mechanisms underlying the pathological manifestations that they display $(1,69)$. Our study addresses mechanisms underlying nonimmune abnormalities of AD-HIES caused by dominant negative mutations in STAT3 $(3,4)$ in order to better understand the disease pathophysiology and find therapeutic targets.

The immunological component of AD-HIES has been the subject of considerable study. Consistent with the critical role that STAT3 plays in signal transduction for many cytokines and growth factors $(5,6)$, previous studies performed on immune cells from AD-HIES patients revealed gross dysregulation of cytokines and chemokines as well as related signaling pathways (3, 4, 29, 30, 35). Experimental trials with hematopoietic stem cell (HPSC) transplantation in these patients have shown some success in the normalization of STAT3 signaling in HPSCs, the normalization of $\mathrm{IgE}$, and the restoration of immune function. However, despite these successes in correcting immune function, nonimmunological abnormalities were not completely corrected (70). The failure to fully correct nonimmunological abnormalities highlights the multisystemic effects of AD-HIES STAT3 mutations in the disease 
A
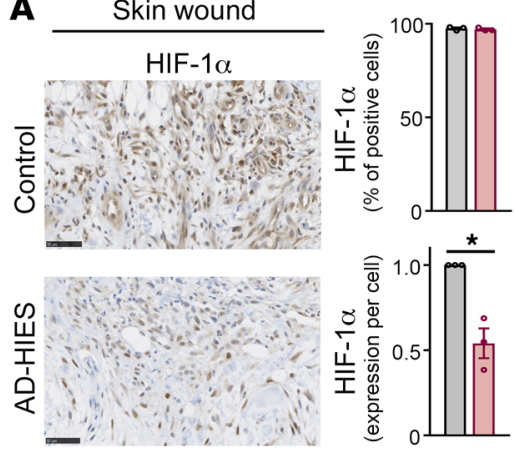

B

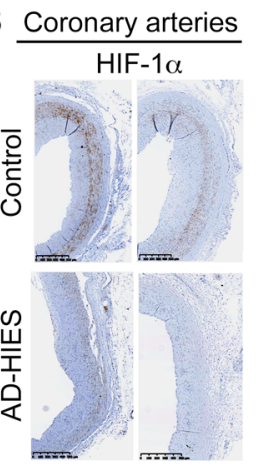

C
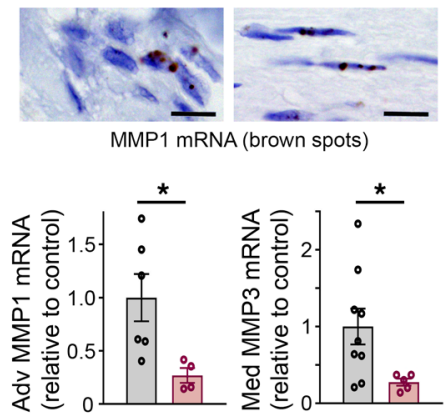

D Adventita vasa vasorum (VV)

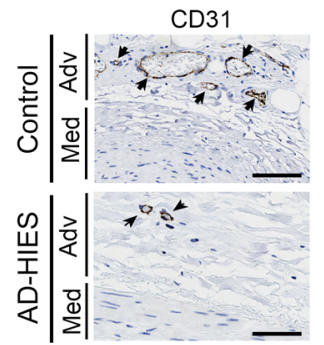

E

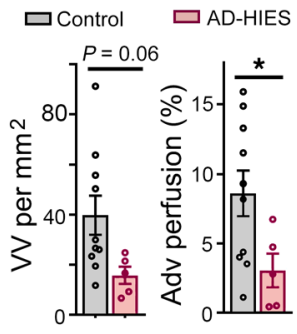

Coronary arteries
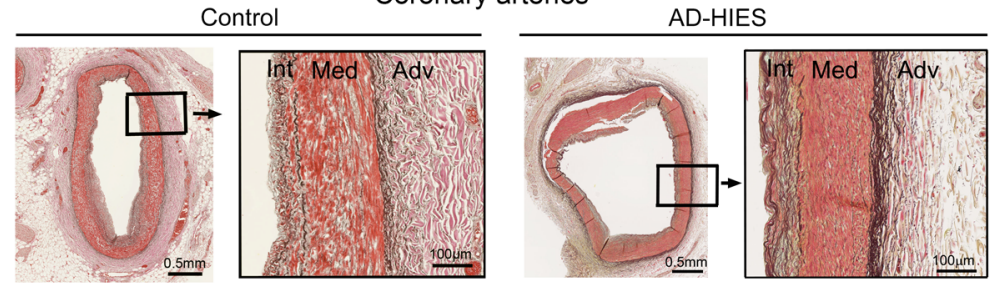

Movat's stain
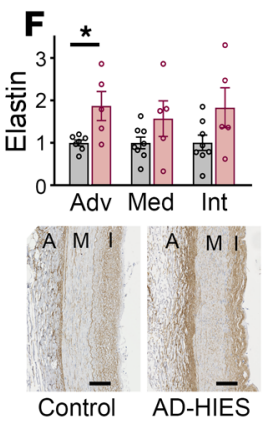

Elastin
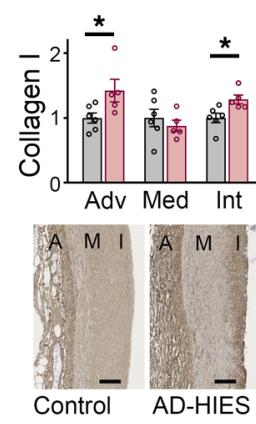

Collagen I
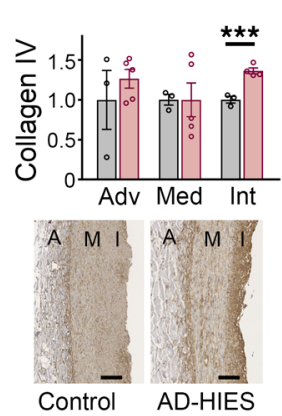

Collagen IV
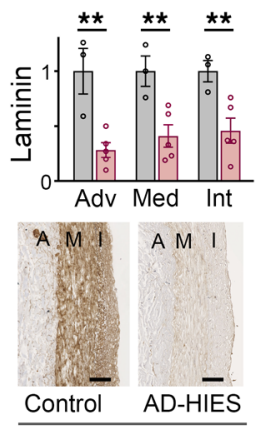

Laminin

Figure 5. Analysis of HIF-1 $\alpha$ expression, vascularization, and ECM composition in skin wounds and coronary arteries of AD-HIES patients. (A) Decreased HIF-1 $\alpha$ expression in granulation tissue during skin-wound healing of AD-HIES patients. Immunostaining of skin biopsy sections 7 days after initial biopsy. See Figure 1 for experiment details. Left: representative images. Scale bars: $50 \mu \mathrm{m}$. Right: quantification (see Methods) $(n=3)$. (B) Decreased HIF-1 $\alpha$ expression in coronary arteries of AD-HIES patients. Immunostaining for HIF-1 $\alpha$ protein. Scale bars: $500 \mu \mathrm{m}$. (C) Decreased levels of MMP1 and MMP3 mRNA in AD-HIES coronary arteries (in situ hybridization RNAscope assay). Top: representative images. Bottom: Quantification of $M M P 1$ ( $n=6$ [control], $n=4$ [AD-HIES]), and MMP3 ( $n=9$ [control], $n=5$ [AD-HIES]) mRNA expression. Scale bars: $10 \mu \mathrm{m}$. See Supplemental Figure 11B for more images. (D) Decreased vasa vasorum perfusion in CA in adventitia. Left: representative images of CA sections stained for CD31 to identify vasa vasorum (black arrows). Scale bars: $100 \mu \mathrm{m}$. Right: image quantification: number of vasa vasorum vessels per $\mathrm{mm}^{2}$ of adventitia and blood perfusion presented as percentages of adventitia area occupied by vasa vasorum ( $n=10$ [control], $n=5$ [AD-HIES]). (E) Increased elastic fibers (black) in adventitia of AD-HIES arteries. (F) Analysis of individual components of ECM by immunostaining with antibodies for elastin, collagen I, collagen IV, and laminin. Bottom: representative images of stainings. Top: quantification. Intensity of DAB staining is measured in the intima, media, and adventitia. Results presented relative to the average intensity in sections from control coronary arteries $(n=3-8)$. Scale bars: $100 \mu \mathrm{m}$. See also Methods and Supplemental Figure 11A for details of analysis. Data are represented as mean \pm SEM. ${ }^{*} P<0.05 ;{ }^{* *} P<0.01 ;{ }^{* *} P<0.001$, 2-tailed unpaired $t$ test. See Supplemental Table 1 for information about patient samples.

pathology and emphasizes the necessity for more studies that target the molecular mechanism beyond the immune system.

In this study, analysis of healing of small skin wounds created by removing cylindrical pieces of skin using a 2-mm punch biopsy tool revealed delayed granulation tissue formation and vascularization in AD-HIES patients (Figure 1). Through RNA-Seq of skin fibroblasts isolated from AD-HIES patients, we identified deficiencies in angiogenesis, ECM metabolism, and wound-healing (Figures 2 and 3) signaling pathways mediated by dysregulation of HIF-1 $\alpha$ signaling (Figure 4). Consistently, HIF-1 $\alpha$ expression was reduced in AD-HIES skin wounds (Figure 5A). We functionally verified these AD-HIES deficiencies in both cell culture and mouse models of angiogenesis: a cell culture vessel tube formation assay, a teratoma growth assay, and mouse HLI and skin-woundhealing models (Figure 3 and Figure 7). We also revealed abnormal organization of the ECM and decreased HIF-1 $\alpha$ expression in coronary arteries of AD-HIES patients, linking vascular structural abnormalities to aberrant STAT3-HIF-1 $\alpha$ signaling (Figure 5). Finally, we explored the possibility of correcting these abnormalities by pharmacological stabilization of HIF-1 $\alpha$ and restoration 
A

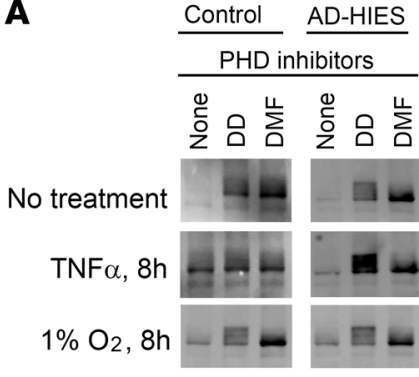

B
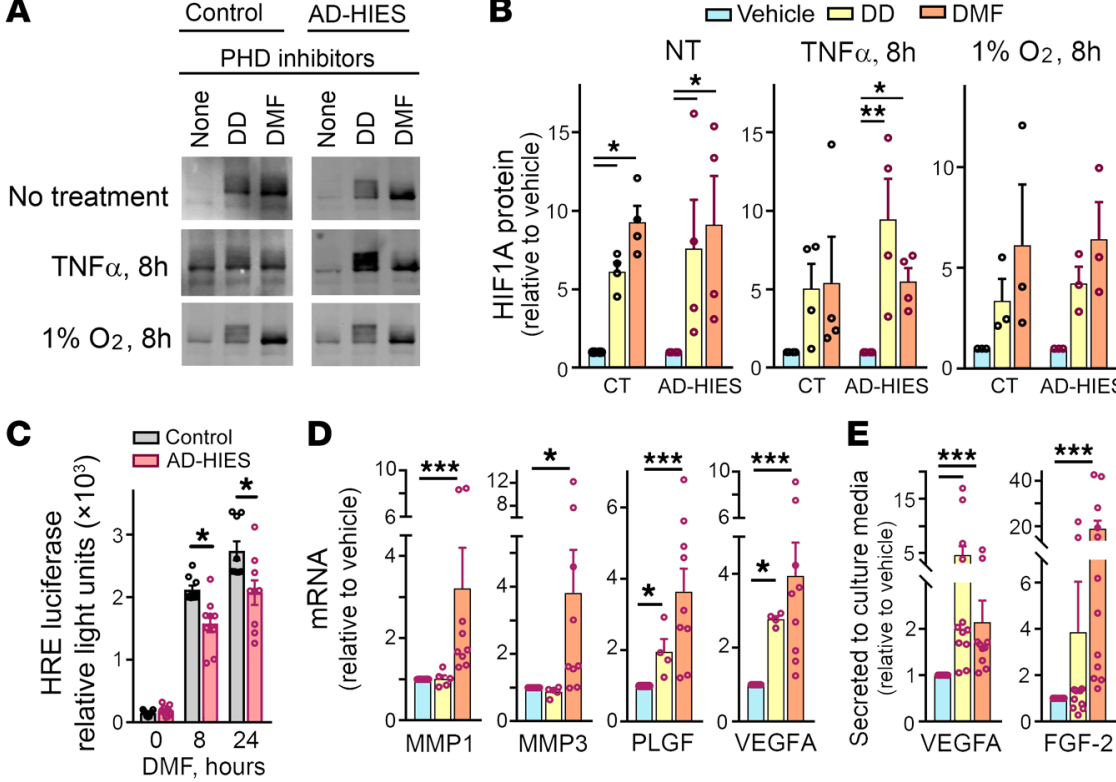

F HUVECs exposed to culture medium collected from AD-HIES fibtoblasts treated with:
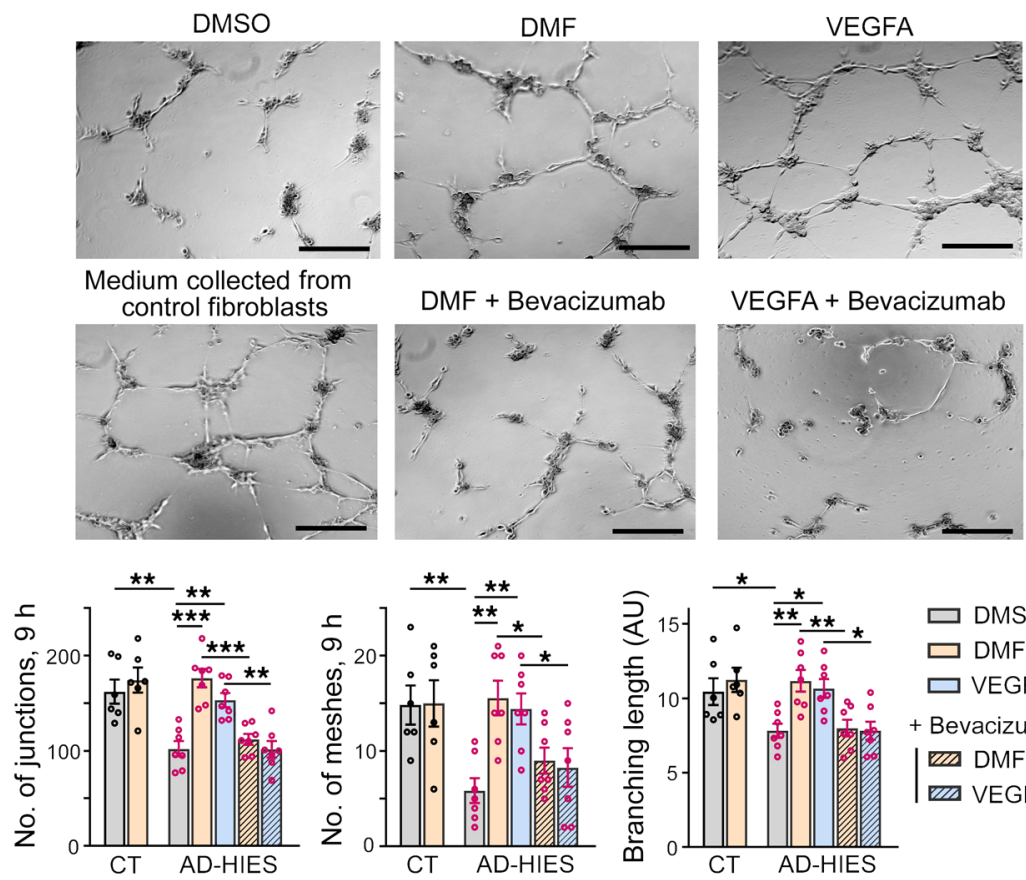

Figure 6. Pharmacological stabilization of HIF-1 $\alpha$ protein increases expression of proangiogenic factors and restores the ability of AD-HIES fibroblasts to support angiogenesis. To stabilize HIF- $1 \alpha$, cells were treated with 2 PHD inhibitors: DMF and daprodustat (DD). (A and B) DMF and DD stabilize HIF-1 $\alpha$ under regular cell culture conditions, under hypoxia and in the presence of TNF- $\alpha$ in AD-HIES fibro-

blasts. (A) Representative Western blot images. (B) Quantification ( $n=3-4)$. (C) DMF increases HIF-1 $\alpha$ transcriptional activity in both control and AD-HIES fibroblasts. Luciferase reporter assay with construct containing HRE was performed under basal conditions $(n=8)$. (D) DMF and DD increase mRNA levels of proangiogenic factors $(n=4-9)$. (E) DMF and $\mathrm{DD}$ increase secretion of proangiogenic factors by AD-HIES fibroblasts (15-hour treatment, $n=11)$. (F) DMF improves ability of cell culture medium collected from AD-HIES fibroblasts to support angiogenesis in an endothelial cell tube formation assay with VEGFA being a major contributor. To neutralize VEGFA, the cell culture medium was supplemented with bevacizumab (see Methods). Top: representative images of HUVECs taken 9 hours after seeding on Matrigel. Scale bars: $200 \mu \mathrm{m}$. Bottom: Quantification of different features of tubule structures ( $n=$ 7). Data are represented as mean $\pm \mathrm{SEM} .{ }^{*} P<0.05$; ${ }^{*} P<0.01$; ${ }^{* *} P<0.001$, 1-way ANOVA followed by Holm-Šidák multiple comparisons test (B and $\mathbf{F}$ ), 2-tailed unpaired $t$ test in (C), Kruskal-Wallis test followed by Dunn's multiple comparisons test (D and $\mathbf{E})$. See Supplemental Table 1 for information about patient samples used. of its signaling (Figure 6 and Figure 7). We demonstrated that stabilization of HIF-1 $\alpha$ protein by PHD inhibitors restores secretion of proangiogenic factors by AD-HIES skin fibroblasts in cell culture (Figure 6) and then showed the efficacy of these inhibitors in improving angiogenesis and skin-wound healing in the mouse model of AD-HIES (Figure 7).

Additionally, recent studies have implicated HIFs in the differentiation of Th17 lymphocytes (56), a key process necessary for normal immune function that is impaired in AD-HIES (68). Indeed, our analysis showed that PHD inhibitors also improve the Th17 differentiation of T cells from AD-HIES patients (Supplemental Figure 13). These results indicate that HIF-1 $\alpha$ stabilization can improve responses to both infection and postinfection healing.
This improvement is particularly impactful in this patient population, given the significant morbidity and mortality stemming from recurrent pulmonary infections and failure to heal effectively afterward (7-9). Excitingly, PHD inhibitors are already being tested in multiple clinical trials for treatment of renal anemia in patients with chronic kidney disease (26). Several of the PHD inhibitors, including daprodustat, which was used in our study, have completed phase II trials and are undergoing phase III clinical development $(27,28)$. Provided outcomes of the trials for the treatment of renal anemia patients are successful, these drugs may become available in the near future to improve deficient postinfection and postsurgery healing in AD-HIES patients, who currently rely only on antimicrobial prophylaxis and supportive multidisciplinary care. 
$\begin{array}{ccc}\text { A } & \text { Left coronary artery (LCA) } \\ \text { WT } & \text { WT } & \text { AD-HIES }\end{array}$

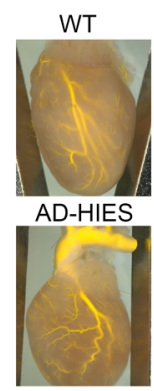

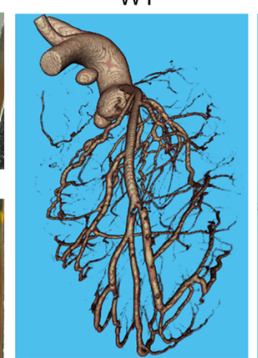

B

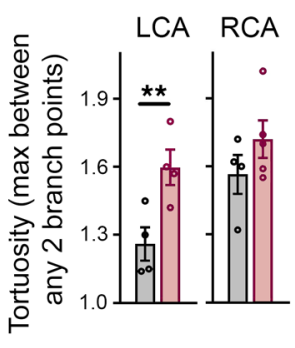

C

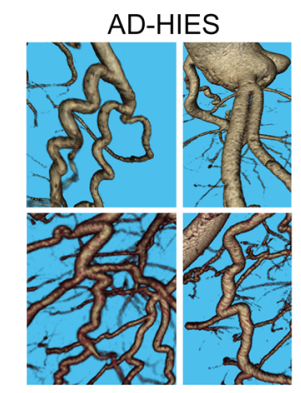

D Mice: $\square$ WT $\square$ AD-HIES

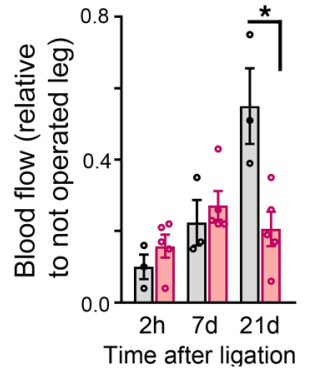

AD-HIES mice gavaged with $\square$ Vehicle $\square$ DMF

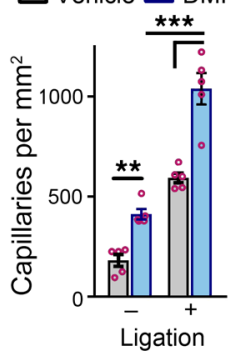

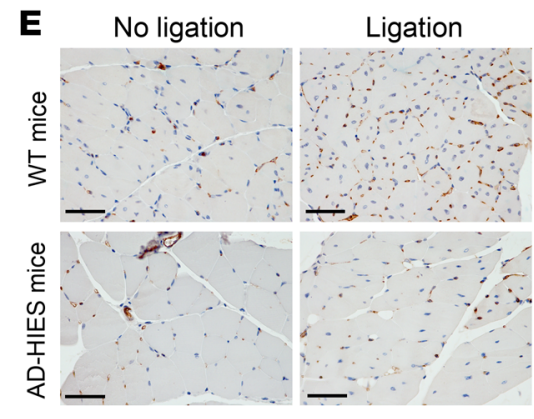

G iPSC injected: 口CONTROL $\square$ AD-HIES
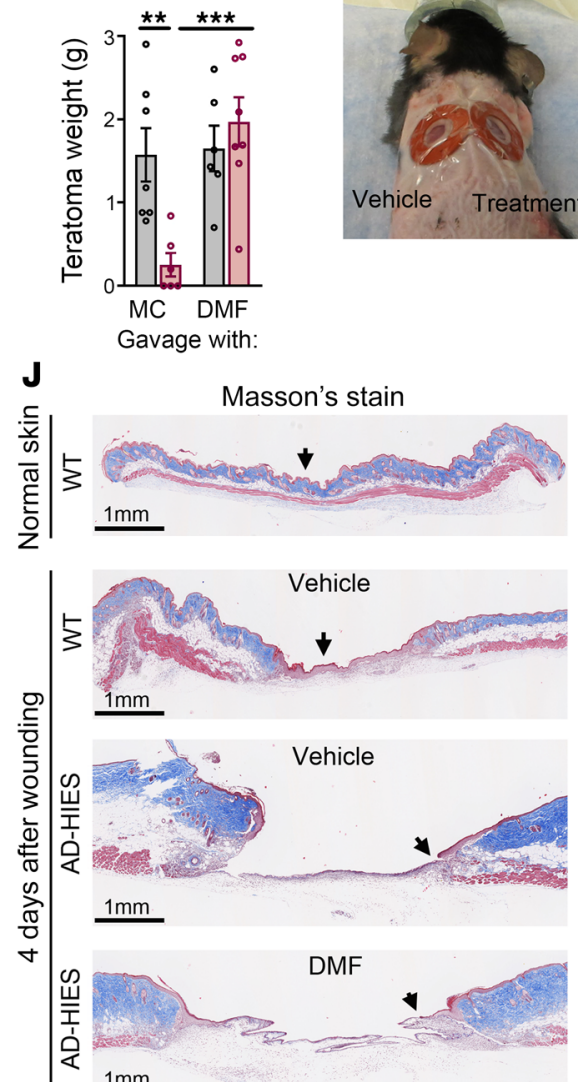
$1 \mathrm{~mm}$
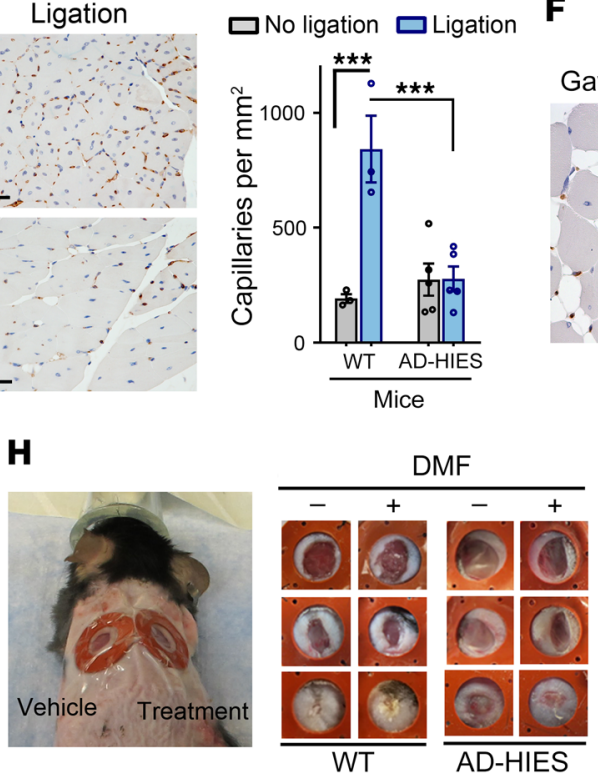

K

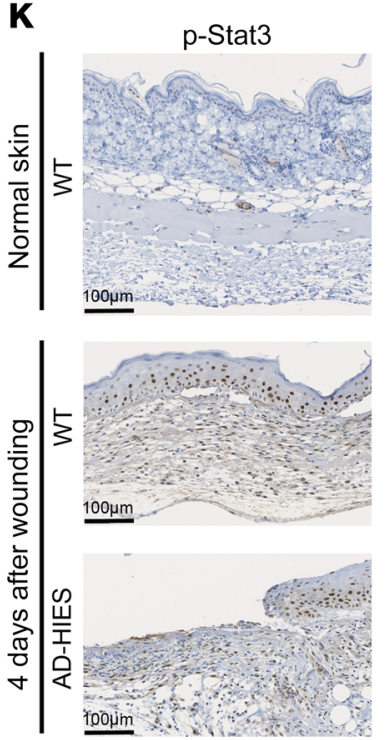

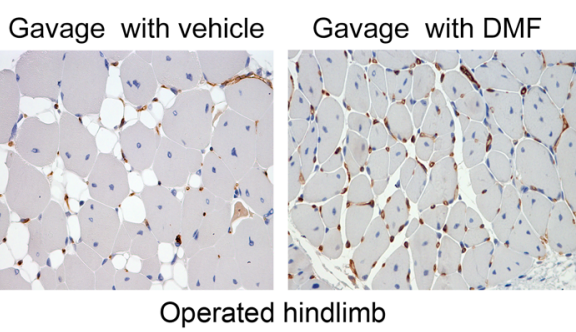

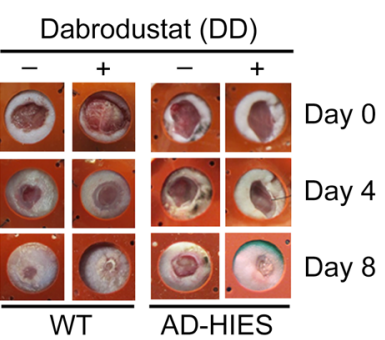

L $\quad$ HIF-1 $\alpha$
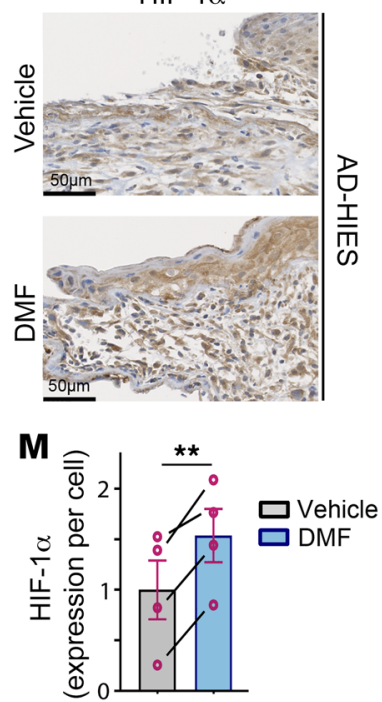

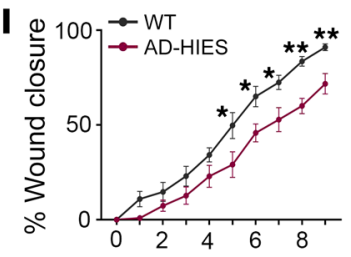

AD-HIES

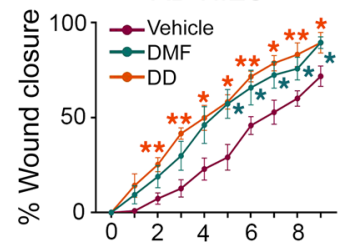

WT

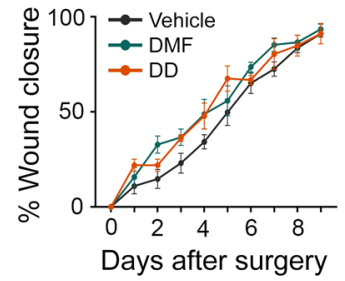


Figure 7. HIF-1 $\alpha$ stabilizers improve angiogenesis and wound healing in AD-HIES mouse model. (A-C) Structural abnormalities of AD-HIES mouse CAs resemble AD-HIES patients. (A) Left: representative images of the hearts. Left CAs (LCAs) filled with silicon are seen. Right: representative images of CA 3D reconstruction. (B) Increased LCA tortuosity $(n=4)$. (C) Examples of abnormalities in secondary branches. ( $\mathbf{D}$ and $\mathbf{E}$ ) Angiogenesis abnormalities in AD-HIES mice: mice failed to restore blood perfusion after ligation of the femoral artery (FA). (D) Quantification by Doppler imaging (WT, $n=3$; AD-HIES, $n=5$ ). (E) Left: Representative images of CD31 staining. Scale bars: $50 \mu \mathrm{m}$. Right: quantification of capillary density $(n=$ 3). (F-M) Stabilization of HIF-1 $\alpha$ by PHD inhibitors improves angiogenesis and wound healing. Treatment with DMF by gavage: $(\mathbf{F})$ improved recapillarization after FA ligation (left: representative images of CD31 staining, original magnification, $\times 100$; right: quantification of capillary densities, $n$ $=5$ ) and (G) restored growth of teratomas from AD-HIES-derived iPSCs ( $n$ = 6). (H-M) Topical treatment with DMF and daprodustat (DD) improved deficient skin-wound healing. (H) Representative images. (I) Quantification of wound closure rates. ( $n=8$ for no-treatment groups; $n=4$ for DMF and DD groups). Slower wound closure in AD-HIES mice was accelerated by DMF and DD. (J-M) Histological analysis of the wounds demonstrating (J) deficient reepithelialization and fibroblast infiltration, (K) increased STAT3 phosphorylation, and ( $\mathbf{L}$ and $\mathbf{M}$ ) increased HIF-1 $\alpha$ expression by DMF treatment. Representative images and quantification of HIF-1 $\alpha$ stainings are shown $(n=4)$. Data are represented as mean \pm SEM. ${ }^{*} P<0.05 ;{ }^{*} P<$ 0.01 ; ${ }^{* *} P<0.001$, 2-tailed unpaired $t$ test (B, D, I, and $\mathbf{M}$ ), 1-way ANOVA followed by Holm-Šidák multiple comparisons test (E-G). See also Supplemental Figure 12 and Supplemental Videos 1 and 2.

The identification of signaling pathways related to ECM metabolism as significantly affected by AD-HIES STAT3 DN mutations (Figure 2B and Supplemental Figure 3) provides an explanation for the many similarities in presentation between AD-HIES patients and patients with other connective tissues disorders, such as Ehlers-Danlos syndromes. These syndromes are caused by genetic variants in gene-encoding collagens and collagen-modifying proteins and, similarly to AD-HIES, present with different degrees of joint hypermobility, tissue fragility, aneurisms, and defective wound healing $(41,42)$.

Taking these data together, our study affirms the fundamental role of STAT3-HIF-1 $\alpha$ signaling in the processes of ECM remodeling, angiogenesis, and wound healing in normal human physiology, explains aberrant wound healing and vascular structural abnormalities in AD-HIES, and identifies HIF- $1 \alpha$ as a potential treatment option for AD-HIES patients. In addition, the study provides in vivo clinical evidence of the importance of STAT3/ HIF-1 $\alpha$ signaling in more common diseases in which tissue remodeling plays a major role, including restrictive lung disease, diabetes, and atherosclerosis.

\section{Methods}

Human subjects. Study subjects were evaluated under a natural history of HIES clinical trial (ClinicalTrials.gov NCT00006150) at the Clinical Center at the NIH. Study subjects were diagnosed with AD-HIES using a diagnostic scoring system comprising immunological and nonimmunological features (71). The diagnosis was confirmed by the identification of STAT3 mutations. Skin biopsies for wound-healing studies were collected under protocols for clinical trial NCT02262819. A description of the AD-HIES patients and control subjects analyzed in this study is given in Supplemental Table 1. Supplemental Table 1 also lists patients and control subjects that were studied in each experiment.
Mice. Two mouse models were used in this study: NSG mice (Jackson Laboratory) and Mut-Stat3 transgenic mice, a mouse model of AD-HIES made on a C57BL/6 background that carries an additional copy of the Stat3 gene with dominant negative AD-HIES mutation (catalog 027952, Jackson Laboratory) (65). Mut-Stat3 mice were a gift from John O'Shea at the National Institute of Arthritis and Musculoskeletal and Skin Diseases (NIAMS)/NIH. Mice were used at ages of 4 to 7 months. Mice were housed in an Association for Assessment and Accreditation of Laboratory Animal Care-accredited facility of the NHLBI. At the end of the experiments, mice were euthanized by $\mathrm{CO}_{2}$ exposure from a compressed source into a closed chamber, followed by cervical dislocation.

See Supplemental Methods for detailed descriptions of the following: human wound-healing model, derivation of patient-specific skin fibroblasts, skin fibroblast cell culture and treatments, derivation and culture of patient-specific HUVECs, RNA-Seq, analysis of RNA-Seq data, RNA extraction and quantification by real-time PCR, Western blot, ChIP, STAT3 overexpression, shRNA knockdown, siRNA knockdown, HRE-luciferase reporter assay, measurement of angiogenic factors in cell culture supernatants, endothelial cell tube formation assay, immunostaining of paraffin-embedded tissue sections, DAB intensity measurements on tissue sections, mouse HLI model, treatment of mice with DMF by gavage, IHC staining for CD31 with DAB visualization for assessment of capillary density, analysis of coronary artery abnormalities in AD-HIES mice, mouse wound-healing model; human coronary arteries, quantification of ECM protein expression on sections of human coronary arteries, quantification of MMP mRNA in sections of human coronary arteries, quantification of the vasa vasorum on sections of human coronary arteries, in vitro differentiation of naive $\mathrm{CD}^{+} \mathrm{T}$ cells from control and $\mathrm{AD}$-HIES patients, teratoma formation from iPSCs and analysis of proportion of $\mathrm{CD} 31^{+}$cells in the teratomas, and immunostaining of teratoma sections for CD31.

Data and materials availability. RNA-Seq data were deposited in the NCBI's Gene Expression Omnibus database (GEO GSE139365). All data needed to evaluate the conclusions in the paper are present in the paper and/or the supplemental materials.

Statistics. Statistical analyses were done using GraphPad Prism 7 and SigmaPlot 13.0 software. All data were tested for normality before the analysis. If the normality test was passed, comparisons were performed by 2-tailed unpaired Student's $t$ test or by 1-way ANOVA followed by Holm-Šidák multiple comparisons test. If not, the comparisons were performed by nonparametric Mann-Whitney $U$ test or by Kruskal-Wallis test followed by Dunn's multiple comparisons test. $P$ values of less than 0.05 were considered significant.

Study approval. The study was approved by the Institutional Review Board of the NIH Clinical Center, NIAID. Written informed consent was obtained from all participants. Mouse studies were approved by the Animal Care and Use Committee of NHLBI. All mouse studies were carried out in strict accordance with the recommendations in the Guide for the Care and Use of Laboratory Animals (National Academies Press, 2011).

\section{Author contributions}

NID designed, performed, and coordinated experiments; analyzed the results; designed and performed RNA-Seq analysis; and wrote the paper. ADW designed and performed the experiments and analyzed the results. DPN and AG performed experiments and analyzed the results. ADW and AG edited the paper. $\mathrm{XZ}, \mathrm{XW}$, and XP analyzed RNA-Seq data. GC designed, led, and 
performed iPSC-derived teratoma experiments. HJ, ZY, and DY performed iPSC-derived teratoma experiments. RS performed mouse experiments. CLD performed sequencing and analysis of HUVEC RNA-Seq. BAK, MDL, RHK, and DL developed and performed mouse coronary artery casting and analysis. JDM, MPO, and DBL designed and performed the Th17 differentiation experiment. ZXY advised on and performed histology staining. IAM ran clinical protocol and took biopsies for the human wound-healing experiment. CCRL performed pathology review of human wound-healing slides. APH, AFF, and SMH cared for patients, provided clinical samples and data, and helped interpret the results. MB conceived the project, designed and performed experiments, analyzed the results, and wrote the paper. Regarding contributions of co-first authors, ADW performed initial key experiments defining the study concept; NID expanded the initial concept and followed up with overall study execution and manuscript writing.

\section{Acknowledgments}

We thank and acknowledge professional skills, advice, and help from the staff of the NHLBI Core Facilities: Jun Zhu at the Sequencing Core for help with RNA-Seq; Philip McCoy, Leigh Samsel, and Ankit Saxena at the Flow Cytometry Core for help with the Luminex assay; and Hong San and James Hawkins at the Animal Surgery and Resources Core for help with mouse studies. We thank staff at the NIH Library Bioinformatics Support Program for providing tools and training for RNA-Seq. We thank Daniil A. Kitchaev for writing and providing Python script for CMYK yellow channel extraction from immunohistochemistry images. We also thank AD-HIES patients and their families for participating in the study. Mut-Stat3 mice were a gift from John O'Shea (NIAMS/NIH). CVPath Institute Inc. provided sections of control coronary arteries. The study was supported by intramural programs of the NHLBI and NIAID.
Address correspondence to: Manfred Boehm, 9000 Rockville Pike, Building 10-CRC, Room 5 East 3132, Bethesda, Maryland 20892, USA. Phone: 301.435.7211; Email: boehmm@nhlbi.nih.gov.

ADW's present address is: University of Colorado Anschutz Medical Campus, Aurora, Colorado, USA.

AG's present address is: Internal Medicine, Department of Medicine, Duke University Hospital, Durham, North Carolina, USA.

XZ's present address is: School of Medicine, Tufts University, Boston, Massachusetts, USA.

XW's present address is: Division of Diabetes, Endocrinology, and Metabolic Diseases, National Institute of Diabetes and Digestive and Kidney Diseases (NIDDK), NIH, Bethesda, Maryland, USA.

HJ's present address is: Department of Hematology, First Affiliated Hospital of Nanjing Medical University, Jiangsu Province Hospital, Nanjing, China.

ZY's present address is: State Key Laboratory of Experimental Hematology, Institute of Hematology and Blood Diseases Hospital, Chinese Academy of Medical Science and Peking Union Medical College, Tianjin, China.

DBL's present address is: Massachusetts General Hospital, Boston, Massachusetts, USA.

JDM's present address is: Division of Allergy, Immunology and Rheumatology, Department of Pediatrics, Columbia University Irving Medical Center, New York, New York, USA.
1. Boycott KM, Ardigó D. Addressing challenges in the diagnosis and treatment of rare genetic diseases. Nat Rev Drug Discov. 2018;17(3):151-152.

2. Degtiar I. A review of international coverage and pricing strategies for personalized medicine and orphan drugs. Health Policy. 2017;121(12):1240-1248.

3. Minegishi Y, et al. Dominant-negative mutations in the DNA-binding domain of STAT3 cause hyper-IgE syndrome. Nature. 2007;448(7157):1058-1062.

4. Holland SM, et al. STAT3 mutations in the hyper-IgE syndrome. $N$ Engl J Med. 2007;357(16):1608-1619.

5. Levy DE, Lee CK. What does Stat3 do? J Clin Invest. 2002;109(9):1143-1148.

6. Yu H, Lee H, Herrmann A, Buettner R, Jove R. Revisiting STAT3 signalling in cancer: new and unexpected biological functions. Nat Rev Cancer. 2014;14(11):736-746.

7. Freeman AF, et al. Lung parenchyma surgery in autosomal dominant hyper-IgE syndrome. JClin Immunol. 2013;33(5):896-902.

8. Freeman AF, et al. Causes of death in hyperIgE syndrome. J Allergy Clin Immunol. 2007;119(5):1234-1240.

9. Freeman AF, Olivier KN. Hyper-IgE syndromes and the lung. Clin Chest Med. 2016;37(3):557-567.
10. Farmand S, Sundin M. Hyper-IgE syndromes: recent advances in pathogenesis, diagnostics and clinical care. Curr Opin Hematol. 2015;22(1):12-22.

11. Chandesris MO, et al. Autosomal dominant STAT3 deficiency and hyper-IgE syndrome: molecular, cellular, and clinical features from a French national survey. Medicine (Baltimore). 2012;91(4):e1-19.

12. Heimall J, Freeman A, Holland SM. Pathogenesis of hyper IgE syndrome. Clin Rev Allergy Immunol. 2010;38(1):32-38.

13. Gharib AM, et al. Coronary abnormalities in hyper-IgE recurrent infection syndrome: depiction at coronary MDCT angiography. AJR Am J Roentgenol. 2009;193(6):W478-W481.

14. Chandesris MO, et al. Frequent and widespread vascular abnormalities in human signal transducer and activator of transcription 3 deficiency. Circ Cardiovasc Genet. 2012;5(1):25-34.

15. Kim Y, et al. Cerebral aneurysm in a 12-yearold boy with a STAT3 mutation (hyper-IgE syndrome). Ann Allergy Asthma Immunol. 2015;114(5):430-431.

16. Falah O, Thwaites SE, Chalmers RT. Ruptured thoracoabdominal aneurysm in a 27-yearold with hyper IgE syndrome. J Vasc Surg. 2012;55(3):830-832.

17. Nussbaum ES, Torok CM, Carroll J, Gunderman
AM. Delayed development of a de novo contralateral middle cerebral artery aneurysm in a patient with hyperimmunoglobulin $\mathrm{E}$ syndrome: a case report. Interv Neuroradiol. 2019;25(4):442-446.

18. Vogel TP, Milner JD, Cooper MA. The ying and yang of STAT3 in human disease. J Clin Immunol. 2015;35(7):615-623.

19. Nieminen $P$, et al. Inactivation of IL11 signaling causes craniosynostosis, delayed tooth eruption, and supernumerary teeth. Am J Hum Genet. 2011;89(1):67-81.

20. Schwerd T, et al. A biallelic mutation in IL6ST encoding the GP130 co-receptor causes immunodeficiency and craniosynostosis. J Exp Med . 2017;214(9):2547-2562.

21. Béziat $\mathrm{V}$, et al. Dominant-negative mutations in human IL6ST underlie hyper-IgE syndrome. JExp Med. 2020;217(6):e20191804.

22. Spencer S, et al. Loss of the interleukin-6 receptor causes immunodeficiency, atopy, and abnormal inflammatory responses. J Exp Med. 2019;216(9):1986-1998.

23. Béziat $V$, et al. A recessive form of hyper-IgE syndrome by disruption of ZNF341-dependent STAT3 transcription and activity. Sci Immunol. 2018;3(24):eaat4956.

24. Frey-Jakobs S, et al. ZNF341 controls STAT3 expression and thereby immunocompetence. $\mathrm{Sci}$ 
Immunol. 2018;3(24):eaat4941.

25. Villarino AV, Kanno Y, O'Shea JJ. Mechanisms and consequences of Jak-STAT signaling in the immune system. Nat Immunol. 2017;18(4):374-384.

26. Maxwell PH, Eckardt KU. HIF prolyl hydroxylase inhibitors for the treatment of renal anaemia and beyond. Nat Rev Nephrol. 2016;12(3):157-168.

27. Del Vecchio L, Locatelli F. Investigational hypoxiainducible factor prolyl hydroxylase inhibitors (HIF-PHI) for the treatment of anemia associated with chronic kidney disease. Expert Opin Investig Drugs. 2018;27(7):613-621.

28. Becker KA, Jones JJ. An emerging treatment alternative for anemia in chronic kidney disease patients: a review of daprodustat. Adv Ther. 2018;35(1):5-11.

29. Yeganeh M, et al. Toll-like receptor stimulation induces higher TNF-alpha secretion in peripheral blood mononuclear cells from patients with hyper IgE syndrome. Int Arch Allergy Immunol. 2008;146(3):190-194.

30. Giacomelli M, et al. SH2-domain mutations in STAT3 in hyper-IgE syndrome patients result in impairment of IL-10 function. Eur J Immunol. 2011;41(10):3075-3084

31. Myles IA, et al. TNF overproduction impairs epithelial staphylococcal response in hyper IgE syndrome. J Clin Invest. 2018;128(8):3595-3604.

32. Kovacic JC, et al. Stat3-dependent acute Rantes production in vascular smooth muscle cells modulates inflammation following arterial injury in mice. J Clin Invest. 2010;120(1):303-314.

33. Subramanian A, et al. Gene set enrichment analy sis: a knowledge-based approach for interpreting genome-wide expression profiles. Proc Natl Acad Sci USA. 2005;102(43):15545-15550.

34. Chehimi J, et al. Cytokine and chemokine dysregulation in hyper-IgE syndrome. Clin Immunol. 2001;100(1):49-56.

35. Zhang Y, et al. PD-L1 up-regulation restrains Th17 cell differentiation in STAT3 loss- and STAT1 gain-of-function patients. J Exp Med. 2017;214(9):2523-2533.

36. Senger DR, Davis GE. Angiogenesis. Cold Spring Harb Perspect Biol. 2011;3(8):a005090.

37. Rousselle P, Montmasson M, Garnier C. Extracellular matrix contribution to skin wound re-epithelialization. Matrix Biol. 2019;75-76:12-26.

38. Ravanti L, Kähäri VM. Matrix metalloproteinases in wound repair (review). Int J Mol Med. 2000;6(4):391-407.

39. Murphy-Ryan M, Psychogios A, Lindor NM. Hereditary disorders of connective tissue: a guide to the emerging differential diagnosis. Genet Med. 2010;12(6):344-354.
40. Visse R, Nagase H. Matrix metalloproteinases and tissue inhibitors of metalloproteinases: structure, function, and biochemistry. Circ Res. 2003;92(8):827-839.

41. Malfait F, et al. The 2017 international classification of the Ehlers-Danlos syndromes. Am JMed Genet C Semin Med Genet. 2017;175(1):8-26.

42. Chiarelli N, Ritelli M, Zoppi N, Colombi M. Cellular and molecular mechanisms in the pathogenesis of classical, vascular, and hypermobile Ehlers-Danlos syndromes. Genes (Basel). 2019;10(8):E609.

43. Gurtner GC, Werner S, Barrandon Y, Longaker MT. Wound repair and regeneration. Nature. 2008;453(7193):314-321.

44. Kano A, et al. Endothelial cells require STAT3 for protection against endotoxin-induced inflammation. J Exp Med. 2003;198(10):1517-1525.

45. Carpenter RL, Lo HW. STAT3 target genes relevant to human cancers. Cancers (Basel) 2014;6(2):897-925

46. Jin $\mathrm{H}$, et al. Increased activity of TNAP compensates for reduced adenosine production and promotes ectopic calcification in the genetic disease ACDC. Sci Signal. 2016;9(458):ra121.

47. Jin $\mathrm{H}$, et al. Generation of human induced pluripotent stem cell lines (NIHTVBi011-A, NIHTVBi012-A, NIHTVBi013-A) from autosomal dominant Hyper IgE syndrome (AD-HIES) patients carrying STAT3 mutation. Stem Cell Res. 2019;41:101586.

48. Couffinhal T, Silver M, Zheng LP, Kearney M, Witzenbichler B, Isner JM. Mouse model of angiogenesis. Am J Pathol.1998;152(6):1667-1679.

49. Limbourg A, Korff T, Napp LC, Schaper W, Drexler H, Limbourg FP. Evaluation of postnatal arteriogenesis and angiogenesis in a mouse model of hind-limb ischemia. Nat Protoc. 2009;4(12):1737-1746

50. Couffinhal $\mathrm{T}$, et al. Impaired collateral vessel development associated with reduced expression of vascular endothelial growth factor in ApoE-/mice. Circulation. 1999;99(24):3188-3198.

51. Schofield CJ, Ratcliffe PJ. Oxygen sensing by HIF hydroxylases. Nat Rev Mol Cell Biol. 2004;5(5):343-354.

52. Semenza GL. Hypoxia-inducible factors in physiology and medicine. Cell. 2012;148(3):399-408.

53. Semenza GL. Oxygen sensing, homeostasis, and disease. NEngl J Med. 2011;365(6):537-547.

54. Eltzschig HK, Carmeliet P. Hypoxia and inflammation. N Engl J Med. 2011;364(7):656-665.

55. Eltzschig HK, Bratton DL, Colgan SP. Targeting hypoxia signalling for the treatment of ischaemic and inflammatory diseases. Nat Rev Drug Discov.
2014;13(11):852-869.

56. Dang EV, et al. Control of T(H)17/T(reg) balance by hypoxia-inducible factor 1 . Cell. 2011;146(5):772-784.

57. Xu Q, et al. Targeting Stat3 blocks both HIF-1 and VEGF expression induced by multiple oncogenic growth signaling pathways. Oncogene. 2005;24(36):5552-5560.

58. Jung JE, et al. STAT3 inhibits the degradation of HIF-1alpha by pVHL-mediated ubiquitination. Exp Mol Med.2008;40(5):479-485.

59. Loboda A, Jozkowicz A, Dulak J. HIF-1 versus HIF-2--is one more important than the other? Vascul Pharmacol. 2012;56(5-6):245-251.

60. Mulligan-Kehoe MJ. The vasa vasorum in diseased and nondiseased arteries. Am JPhysiol Heart Circ Physiol. 2010;298(2):H295-H305.

61. Abd-Elmoniem KZ, et al. Coronary atherosclerosis and dilation in hyper IgE syndrome patients: Depiction by magnetic resonance vessel wall imaging and pathological correlation. Atherosclerosis. 2017;258:20-25.

62. Wagenseil JE, Mecham RP. Vascular extracellular matrix and arterial mechanics. Physiol Rev. 2009;89(3):957-989.

63. Min JH, Yang H, Ivan M, Gertler F, Kaelin WG, Pavletich NP. Structure of an HIF-1alpha -pVHL complex: hydroxyproline recognition in signaling. Science. 2002;296(5574):1886-1889.

64. Bruick RK, McKnight SL. A conserved family of prolyl-4-hydroxylases that modify HIF. Science. 2001;294(5545):1337-1340.

65. Steward-Tharp SM, et al. A mouse model of HIES reveals pro- and anti-inflammatory functions of STAT3. Blood. 2014;123(19):2978-2987.

66. Park SA, et al. Full-thickness splinted skin wound healing models in $\mathrm{db} / \mathrm{db}$ and heterozygous mice: implications for wound healing impairment. Wound Repair Regen. 2014;22(3):368-380.

67. Wang X, Ge J, Tredget EE, Wu Y. The mouse excisional wound splinting model, including applications for stem cell transplantation. Nat Protoc. 2013;8(2):302-309.

68. Milner JD, et al. Impaired T(H)17 cell differentiation in subjects with autosomal dominant hyperIgE syndrome. Nature. 2008;452(7188):773-776.

69. Fishman MC. Power of rare diseases: found in translation. Sci Transl Med. 2013;5(201):201ps11.

70. Yanagimachi M, et al. The potential and limits of hematopoietic stem cell transplantation for the treatment of autosomal dominant hyper-IgE syndrome. J Clin Immunol. 2016;36(5):511-516.

71. Woellner C, et al. Mutations in STAT3 and diagnostic guidelines for hyper-IgE syndrome. JAllergy Clin Immunol. 2010;125(2):424-432.e8. 